\title{
Butterfly Diversity from Farmlands of Central Uganda
}

\author{
M. B. Théodore Munyuli ${ }^{1,2,3}$ \\ ${ }^{1}$ Department of Biology and Environment, National Center for Research in Natural Sciences, CRSN-Lwiro, D.S. Bukavu, \\ Kivu, Democratic Republic of Congo \\ ${ }^{2}$ Département de Nutrition et Dietetiques, Centre de Recherche pour la Promotion de la Santé, \\ Institut Supérieur des Techniques Médicales, ISTM Bukavu, Sud-Kivu, Democratic Republic of Congo \\ ${ }^{3}$ Department of Environmental Management, College of Agricultural and Environmental Sciences, Makerere University, \\ P.O. Box 7062, Kampala, Uganda
}

Correspondence should be addressed to M. B. Théodore Munyuli, tmunyuli@yahoo.com

Received 9 October 2011; Accepted 31 May 2012

Academic Editor: Robert Matthews

Copyright ( $\odot 2012$ M. B. Théodore Munyuli. This is an open access article distributed under the Creative Commons Attribution License, which permits unrestricted use, distribution, and reproduction in any medium, provided the original work is properly cited.

The aim of this study was to collect information about the diversity of butterfly communities in the mixed coffee-banana mosaic (seminatural, agricultural) landscapes of rural central Uganda. Data were collected for one year (2006) using fruit-bait traps, line transect walk-and-counts, and hand nets. A total of 56,315 individuals belonging to 331 species, 95 genera, and 6 families were sampled. The most abundant species was Bicyclus safitza (14.5\%) followed by Acraea acerata (6.3\%), Catopsilia florella (6.5\%) and Junonia sophia (6.1\%). Significant differences in abundance, species richness, and diversity of butterflies occurred between the 26 study sites. Farmland butterflies visited a variety of habitats within and around sites, but important habitats included woodlands, fallows, hedgerows, swampy habitats, abandoned gardens, and home gardens. The highest diversity and abundance of butterflies occurred in sites that contained forest remnants. Thus, forest reserves in the surrounding of fields increased the conservation values of coffee-banana agroforestry systems for butterflies. Their protection from degradation should be a priority for policy makers since they support a species-rich community of butterflies pollinating cultivated plants. Farmers are encouraged to protect and increase on-farm areas covered by complex traditional agroforests, linear, and nonlinear seminatural habitats to provide sufficient breeding sites and nectar resources for butterflies.

\section{Introduction}

Butterflies are considered as good ecological indicators for other invertebrate taxa [1-3] and as surrogate representatives of environmental quality changes. Butterflies play significant ecological roles in agricultural landscapes. They perform essential ecosystem services [4], especially in the recycling of nutrients (N, P, K) highly needed by crops that were previously taken through plant absorption and uptake. Their larval stages feed on leaves of several wild plants found in the agricultural systems and therefore release their faeces that contain some amount of nutrients [5]. In addition, butterflies are food to birds and other predators and are hosts to several parasitoids that suppress crop pests [6].

Despite their diversity, ubiquity, and importance particularly with regard to their ecology, behavior, and functional role (e.g., pollination activities), butterflies remain relatively little studied in farmland habitats [5] in the tropics. In agricultural systems, several butterfly species are suspected to be important pollinators of wild and cultivated crop species on which human beings depend on for their livelihoods [7].

Among the invertebrates, butterflies are one of the best studied insect groups for which both ecological and relatively good quantitative distribution data are available worldwide. In Mediterranean countries [8], knowledge of butterflies inhabiting farmland habitats is fairly good compared to most of the regions of sub-Sahara Africa where the knowledge is poor or absent.

Roughly $90 \%$ of butterfly species live in the tropics [7]. Despite this, very little is known about tropical farmland butterfly ecology particularly when compared to temperate butterfly systems. The relative scarcity of data on 
tropical butterfly species hampers the ability to effectively conserve them, particularly as pollinating agents in agricultural systems.

In Uganda, most studies on butterflies have been carried out in natural areas, forest ecosystems, and in protected areas [9-12]. There is a lack of empirical data on butterflies from farmland habitats in sub-Sahara Africa and in Uganda. No published data exists describing the diversity of butterflies found in agricultural landscapes in Uganda. However, such information is important for butterfly biodiversity and ecosystem services conservation on farmlands.

Because butterflies provide important ecological services for crops and native wild plant species in many ecosystems of the world [13], their conservation is essential to sustain the productivity of natural and agricultural landscapes. However, the protection, conservation, and utilization of Lepidoptera pollinator diversity require extensive understanding of their foraging behaviours and of their temporal and spatial distribution in agricultural landscapes [14, 15]. Farmland habitats support a rich and functionally diverse butterfly community.

Preliminary field observations conducted in central Uganda [7] indicated that some butterfly species are present and busy collecting floral resources in vegetable and legume crop fields later in the morning hours to evening time. Although a proper assessment of pollination efficiency of different species has not been conducted, the abundance and long visits to crop flowers indicated that some of these crops were receiving butterfly pollination services [7]. Preliminary observations indicated also that the annual contribution of butterflies to total pollination services delivered to crops in Uganda is in the range from $4.0 \%$ to $9.0 \%$ [7]. Thus, butterflies may constitute the second group of crop pollinators behind bees in central Uganda [7]. It is likely that some species are both good pollinators of some cultivated and wild plant species. There is a need to conserve butterflies in rural landscapes of central Uganda.

The aim of this study was to collect information on the current status of butterflies in farmlands of central Uganda. The specific objectives were (i) to characterize butterfly assemblages (abundance, species richness and diversity) on farmlands of central Uganda, (ii) to compare butterfly assemblages in sites with different seminatural habitats/landuses and farm management systems, and to identify environmental factors (land-uses, habitats) mostly determining the distribution and occurrence of different species in the farmlands of central Uganda.

In this study, it was hypothesized that farmlands do not support diverse butterfly communities. It is predicted that butterfly assemblages on farmlands in sites with different land-uses, seminatural habitats and farm management systems in central Uganda are of similar taxa given the farmland character that is over-cultivated.

\section{Material and Methods}

2.1. Study Area. This study was conducted in the bananacoffee system of Lake Victoria Arc covering several districts of the central Uganda (Figure 1). The study zone is characterized by ferrisoils with high to medium fertility level and receives on average $1000-1800 \mathrm{~mm}$ of rains on a bimodal pattern (rainy seasons: March-May, September-November; semi-dry to dry seasons: June-August, December-February) with $22-28^{\circ} \mathrm{C}$ and $60-75 \%$ of mean annual temperature and relative humidity respectively [7]. The study zone is farmland area found around Lake Victoria. The farmland zone is characterized with shrubs of Acacia spp., legume trees, melliferous plant species, Papyrus and palms ranging from 2 to $15 \mathrm{~m}$ high dominating the remnant secondary vegetation.

Several food and cash crops are grown, mainly cassava (Manihot esculentum L.), sweet potato, (Ipomoea batatas L.), maize (Zea mays), beans (Phaseolus vulgaris L.), groundnut (Arachis hypogea L.), tomato (Lycopersicon esculentum), watermelon (Citrullus lanatus), pumpkin (Cucurbita moschata), cucumber (Cucumis sativus), melon (Cucumis melo), chilies (Capsicum spp.), and several other fruits, vegetables and horticultural crops (cabbage, onion, egg plants, sim-sim, etc.). The majority of these crops are grown in small-scale monoculture and/or polyculture fields that are integrated into the coffee-banana agroforest production systems where coffee and banana are the major crops.

Rural central Uganda is mosaic landscape where "islands" of patches of natural habitats (forest reserves, wetlands, woodlands) are found scattered within agricultural matrices dominated by linear and nonlinear features of seminatural habitats (fallows, hedgerows, grasslands, woodlots, rangelands) that serve as field boundaries for a diversity of small-scale fields. Compared to other regions of Uganda, central region is characterized by high demographic pressure, limited access to arable lands, continuous cultivation, and overexploited lands.

This study was conducted at 26 study sites that were previously selected to represent a range of land-use types of varying degrees of anthropogenic disturbances and farm management intensities. Thus, all study sites were characterized by the presence of contrasting habitat and landuse types in the vicinity. The different study sites were grouped into clusters such as each cluster contained 2 to 4 study sites. The different study sites were separated from one another by a distance from 5 to $50 \mathrm{~km}$. The different study sites were later regrouped into different farm management systems (modernized large-scale fields, traditional small-scaled fields). They were also grouped into clusters of different land-use intensity gradients or categories (low, medium, high, very high). Detailed description of the different land-use intensity classes occurring in the study area are presented in Munyuli [7]. Characteristics of dominant agroforestry systems found in central Uganda are presented in Table 1.

2.2. Field Sampling of Butterflies. Field sampling of butterflies was conducted at 26 study sites. In each of the 26 study sites, an area of $1 \mathrm{~km}^{2}$ was selected and the area was divided into five linear parallel transects of $1000 \mathrm{~m}$ long and $200 \mathrm{~m}$ apart (Figure 2). Transects were used as basic sampling 


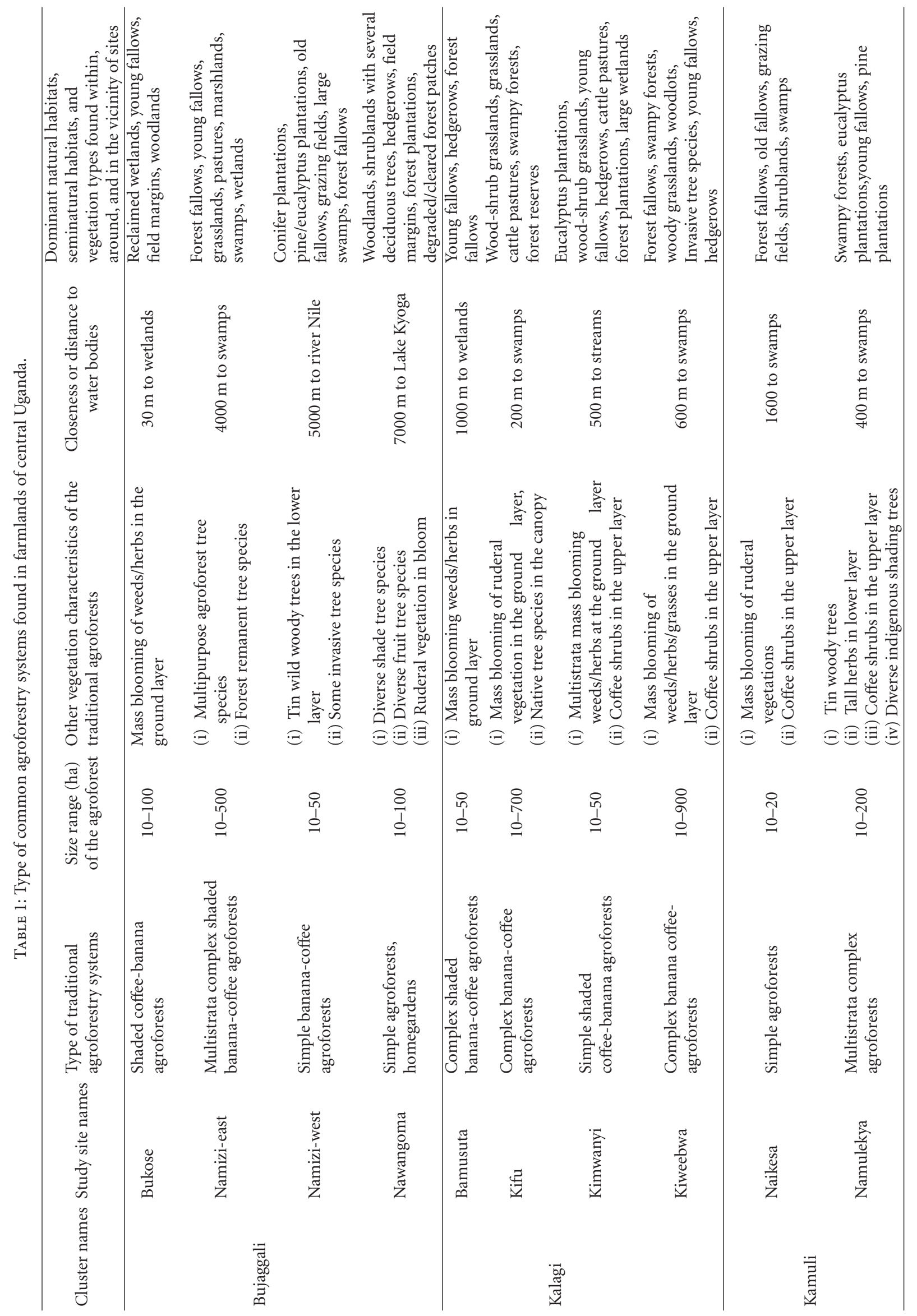




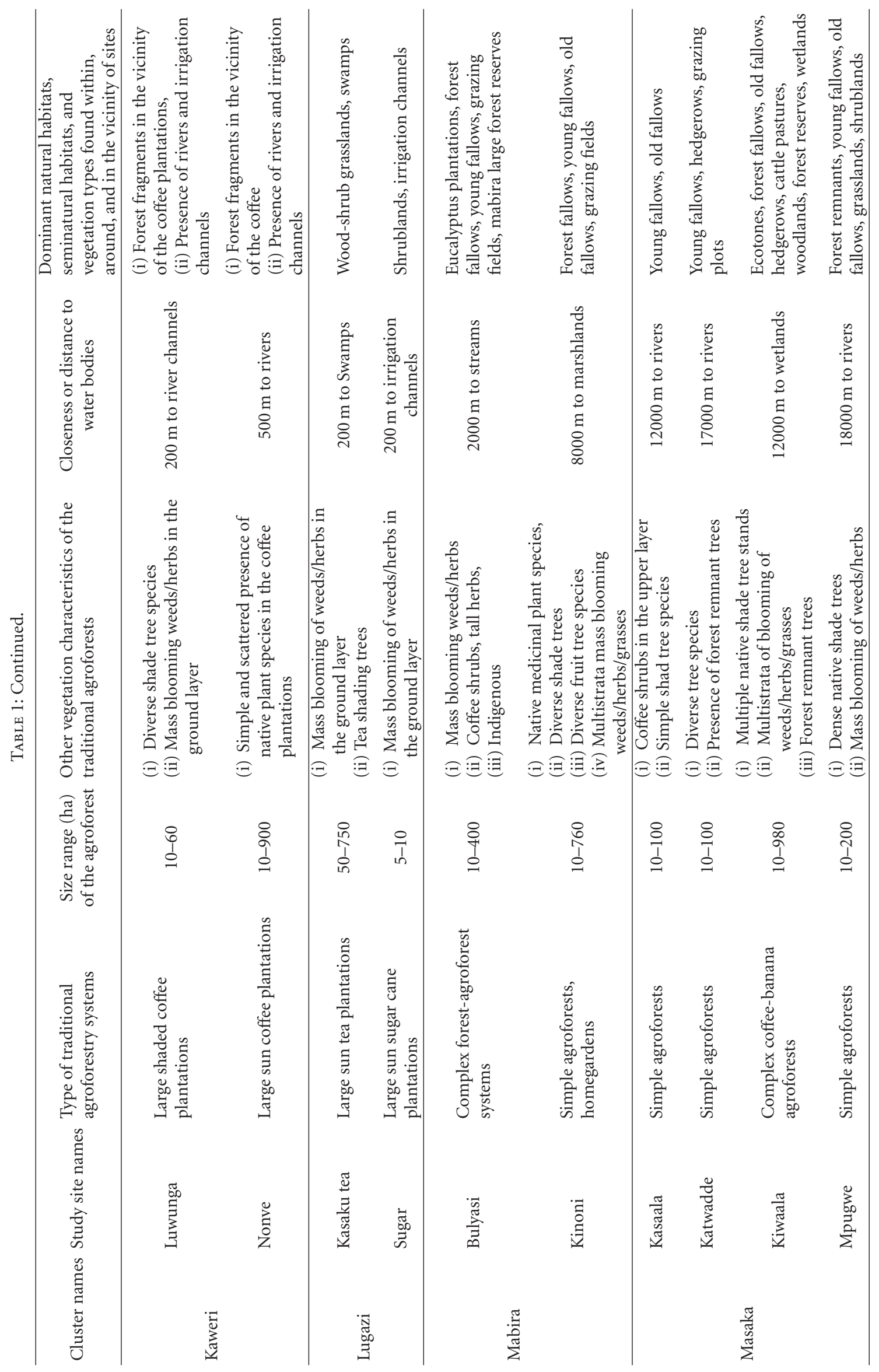




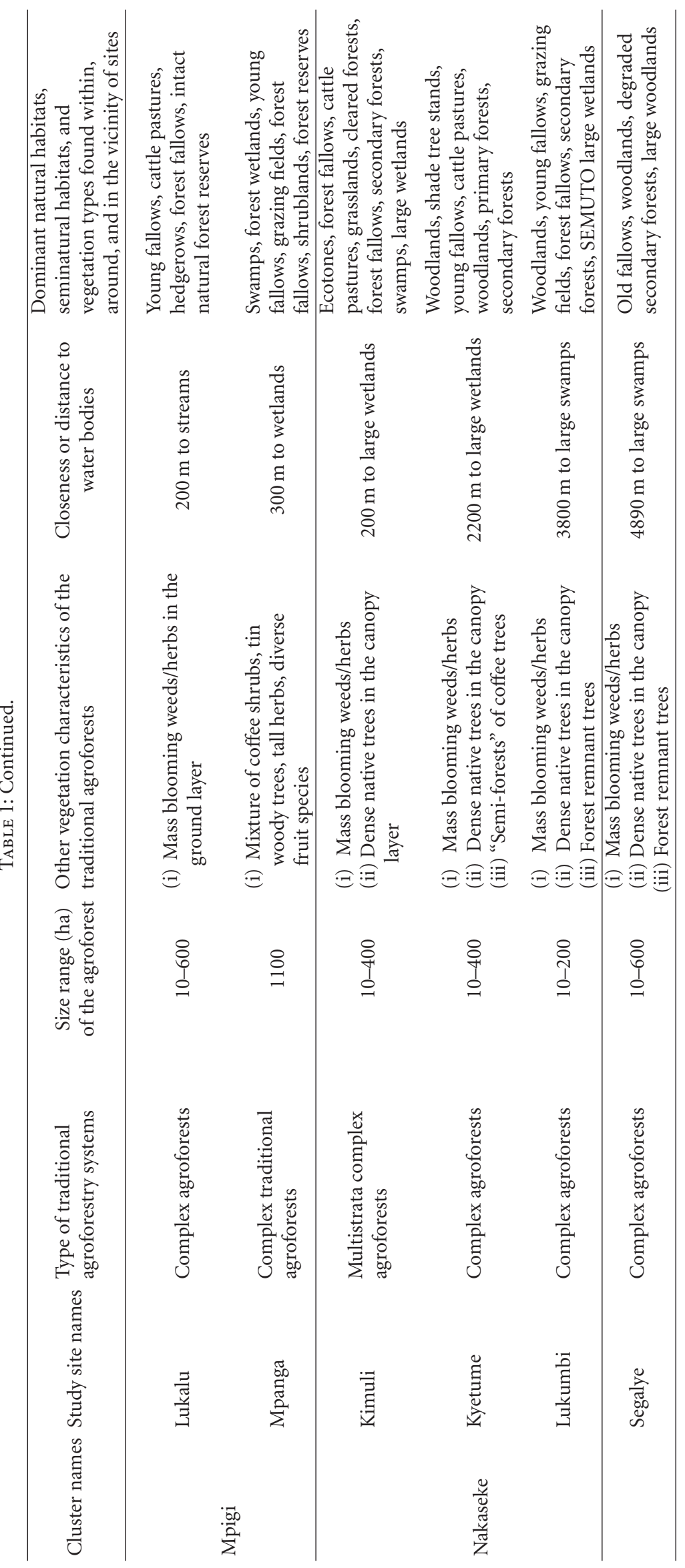




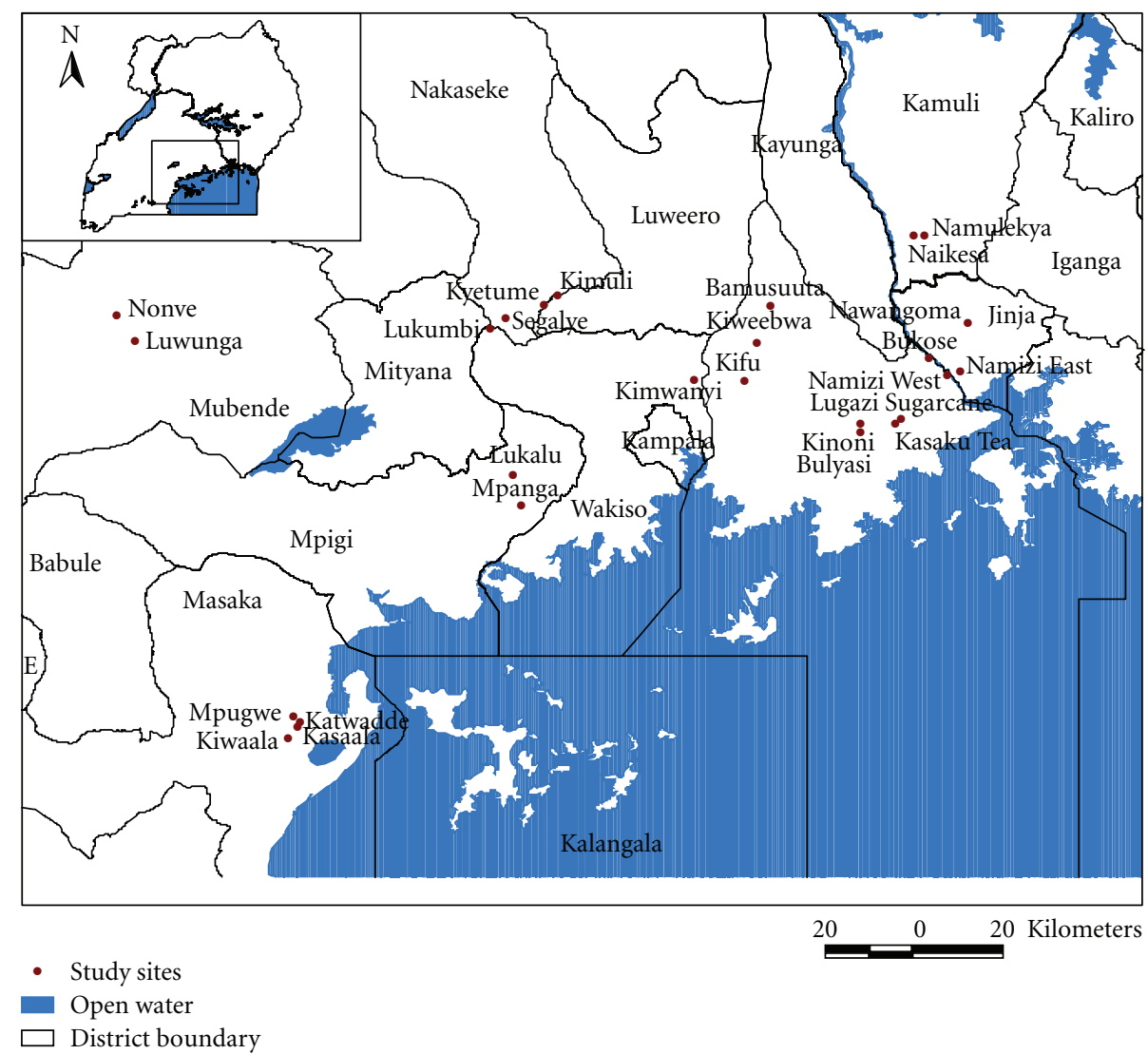

FIGURE 1: Location of districts and study sites from the banana-coffee farming system located around Lake Victoria in Uganda.

unit for all butterflies, land-uses, seminatural habitats, and vegetation data. Thus, butterflies were sampled on one central transect per study site due to time and resource constraints and due to the high number of sites targeted to be sampled during every data collection round. The central transect was selected to represent different seminatural habitats, vegetation, and land-use types of transects that were not sampled. The selected transect was surveyed for five rounds of data collection. A "sampling belt" (20 m wide $\times$ $1000 \mathrm{mlong}=2 \mathrm{ha}$ ) or "sampling plot" was selected in the middle of each central transect [7]. The size of the sampling plot was fixed based on author field experience. The "sampling plot" was kept constant across all sampling dates conducted in each study site.

Each sampling period, the sampling was conducted by two surveyors (the author and one research assistant). To reduce on biases and obtain confidential data, the research assistant was previously trained in butterfly sampling techniques. To assess data reliability, the repeated counts per transect were the same on each sampling date. Using each of the three sampling methods above mentioned, butterflies were sampled during five consecutive rounds from January 2006 to December 2006 (Round 1: January-April, Round 2: May-June, Round 3: July-August, Round 4: SeptemberOctober, and Round 5: November-December) in each of 26 sites studied. During sampling periods, weather data in the different study sites were similar across rounds. Sampling was conducted across crop growing seasons in order to enable further comparisons of results between rainy (wet) and dry seasons. Butterflies were sampled under good weather conditions during sunny and calm days from 09 h00 to $17 \mathrm{~h} 00$.

Overall, butterflies were sampled using three complementary methods (transect walk-and-counts, hand nets, and fruit bait traps) universally recommended and extensively used to survey and monitor butterfly populations and communities [16-18] in the tropics. These methods have been applied in Uganda in previous studies [19-21]. Details of the three sampling methods applied are presented in Munyuli [5]. The different sampling methods are hereby briefly presented.

2.2.1. Transect Walk-and-Counts. Butterfly communities were counted using line transect method also called "visual census method" [7]. During each sampling visit, butterflies were counted while walking at a steady pace of $5-10 \mathrm{~m} / \mathrm{min}$ along transect lines, frequently stopping, and observing butterfly species within transect range. With experience and ability to identify correctly as many species as possible by observers/recorders, butterflies were identified on the wing (using wing characteristics) while flying out and the total number of butterflies of each species flying within view of the observer was recorded. Butterflies that could 


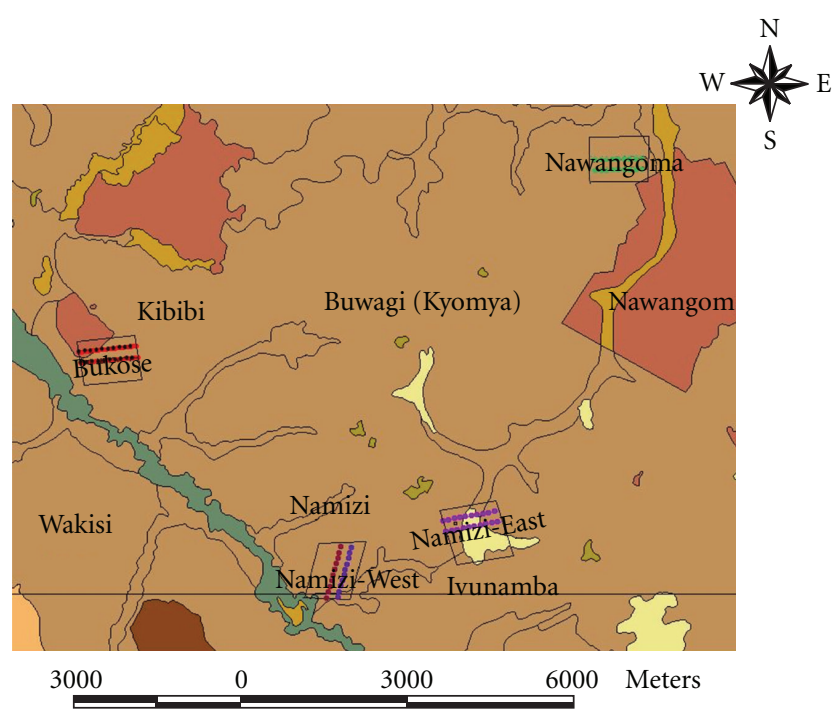

- Nawangoma points

$\checkmark$ Nawangoma transects

Nawangoma study site

Namizi-east site

- Namizi-east points

$\checkmark$ Namizi-east transects

- Namizi-west points

- Namizi-west points

Namizi-west transects

Namizi-west study site

Bukose study site

- Bukose sampling points

Bukose sampling transect

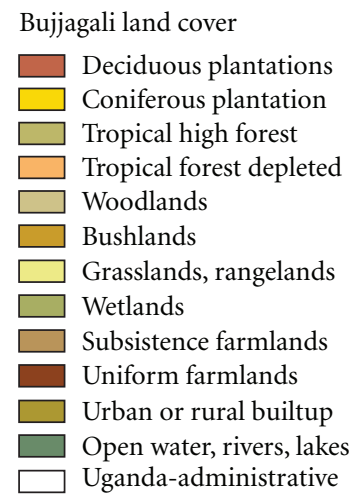

Figure 2: Location of Bukose, Namizi-east, Namizi-west, and Nawangona study sites in Jinja district.

not be identified on spot were captured for laboratory identification. While sampling to both sides of the transect, caution was taken to sufficiently avoid double counting or recounting of individuals of a given species by walking in one direction and by not moving back to resample a species seen behind the surveyor. To avoid counting individuals and species more than once, they were released far away from the sampling belt (transect). Thus, during transect walks, specimens were not collected.

2.2.2. Hand-Netting Method. Hand-netting was carried out immediately after visual counts were finished, using an ordinary insect hand net. Hand-netting was conducted for 20 to 30 min per transect and involved sampling ("handnetting") all butterflies that could not be identified on-spot during visual censuses, at every patch of nectarine plants that could be found alongside the transect. Captured butterflies were counted. Majority of captures were released after field identification, doubtful specimens were collected in paper packets and taken to the laboratory for proper identification.
2.2.3. Banana Fruit-Bait Traps Method. Butterflies were captured in traps baited with mashed ripe bananas that had been fermenting in plastic buckets for 2 days. The bait was prepared three days prior to baiting and not replaced unless lost. Traps were made with local materials, based on the VanSomeron-Rydon trap design $[12,21]$. Thus, the traps were cylinders of white nylon netting $(125 \mathrm{~cm}$ high and $35 \mathrm{~cm}$ in diameter) sewn onto a frame of two metal rings, closed at the top and open at the bottom. The trap nets were $125 \mathrm{~cm}$ in height, minimizing the risk of escape once butterflies had entered. Traps were placed at about $100 \mathrm{~m}$ interval along transect lines. At each trap point, one trap was placed in sunny area while the other was hanged in a shaded habitat to maximize the chance of trapping species attracted by different habitat management in the agricultural landscapes. Each trap baited with two spoons of fermenting banana, was suspended $1.5-2 \mathrm{~m}$ above the ground. Traps placed in sunny or shaded environments were hanged on trees and shrub branches using a slingshot and weighted fishing line. Traps were installed preferably in the evenings between $16 \mathrm{~h} 00$ and $18 \mathrm{~h} 00$ and samples removed after $48 \mathrm{~h}$. Overall, twentybaited traps were set each sampling visit in each study site. During the untrapping process, butterflies in the traps were counted and few individuals of the same species were collected in glassine paper envelopes for identification in the laboratory at Makerere University, the rest of the specimens found alive were released after they being recorded.

Butterfly specimens were identified by consulting literature, nomenclature, and colored plates of butterflies and by using the reference collection available at Makerere University Zoology Museum. The taxonomic characteristics of butterflies were obtained from standard guides including (i) Butterflies of Kenya [22]; (ii) "Butterflies of West Africa" [23]; (iii) "Butterflies of Uganda" [24]. Identification of all voucher specimens was confirmed by a butterfly taxonomist (Mrs. Akite Perpertra) based at Makerere University Zoology museum. A collection of butterfly voucher specimens from the 26 study sites is deposited at the Zoology Museums, Makerere University. Butterfly collection permit in farmlands of Uganda was issued by the Uganda national council of Science and technology (UNCST). Butterflies were collected under an officially registered project in Uganda (Darwin project reference: 14-032).

2.3. Environmental Factors. A variety of "environmental factors" (land-uses, seminatural habitat, and natural habitats) are used as foraging/breeding habitats by diverse butterfly species in central Uganda. These "environmental factors" are likely influencing occurrence of different foraging butterfly species on transects. Concurrently with butterfly surveys, different land-uses and seminatural habits that met within and in the vicinity of crop fields during transect walks were noted every sampling day in each study site. The size (ha) of the habitat was also recorded simultaneously to the registration of butterfly species and individuals visiting nectarine patches within the habitat/land use.

During transect walks, activities of different butterfly species on blooming crops were noted. In each study site 
and in each habitat/land use, the number of plant species in bloom was recorded each sampling day in order to estimate the availability of nectarine resources for butterflies on a monthly basis. In addition, average climatic data (rainfall, temperature) for year 2006 was calculated using raw data from 4 meteorological stations located in the study zone for year 2006. Detailed information on meteorological data from the study region can also be obtained from the office of the prime minister, government of Uganda, meteorological department, Kampala, Uganda [7].

2.4. Butterfly Biodiversity Analysis. For the basic biodiversity analysis, the data from the three sampling methods were pooled to obtain total butterfly biodiversity per 2 ha transect per study site per sampling round as recommended [7]. In addition to personal field observations, data on butterfly functional groups were obtained from published records. The nomenclature of butterfly habitat associations and ecological group was adopted from Larsen $[22,23]$ and from Davenport [25].Thus, every species recorded was assigned to one of the following ecological habitat categories: $\mathrm{F}=$ forestdependent species; $\mathrm{f} / \mathrm{m}=$ forest edge/woodland habitats and migratory specialist species; $\mathrm{f}=$ forest edge/woodland species; $\mathrm{O}=$ open habitat specialist species; $\mathrm{O} / \mathrm{m}=$ open habitat/migratory species; $\mathrm{S}=$ swamp/wetland specialist species; $\mathrm{W}=$ widespread species; $\mathrm{U}=$ species of unknown (undescribed) habitat preference; $\mathrm{W} / \mathrm{m}=$ widespread species and migratory species; $\mathrm{m}=$ migratory species. The proportion of the $\mathrm{F}$ and $\mathrm{f}$ species in a sample is an indication of the ecological state (habitat of good quality) of the habitat when working in forest habitats [19] and in similar agricultural matrices. For each ecological category, species richness and abundance were calculated using pooled data from the three sampling methods.

For the biodiversity analysis, species dominance $(D)$ was calculated according to Buschni and Woiski [26]: $D=$ (abundance of a species/total abundances recorded) $\times 100$. If $D>5 \%$, the species was termed a dominant species; if $2.5 \%<D<5 \%$, the species was termed an accessory species or species of intermediate abundance, and if $D<$ $2.5 \%$, the species was termed as an incidental species. Rare species (species represented by less than 5 individuals and or sampled from only one site) and unique species (species occurring with one individual: singleton, or with two individuals: doubleton) were noted whenever present per sample obtained each sampling round as recommended $[26,27]$.

Species richness was estimated by considering the number of butterfly species recorded each sampling round [27]. Total species recorded per study site was used to get the average since the interest was to detect the pattern. Butterfly faunal diversity was estimated by calculating the Evenness and the Shannon diversity indices as recommended [27].

Species accumulation curves, which plot the cumulative number of species (S) as a function of sampling effort, were used as recommended [27]. To determine the degree to which the sampling effort and protocol accurately reflected the butterfly community, species accumulation curves were generated based on continuous samples obtained in each round of data collection as recommended [27]. Species accumulation and estimation curves were generated using the Jacknifel estimator. Jacknife-1 was calculated using Microsoft Excel 2003 computer program following approaches described by [27].

The Sorensen similarity index $[27,28]$ was used to compare similarities among sampling methods. Sørensen's index of similarity (IS) was calculated using the following equation: IS $=[(2 C / 2 C+A+B)] \times 100$, where $C$ is the number of species in common between two treatments having $A$ and $B$ number of species, respectively. An IS value of 100 indicates that the two treatments contain the same number of species (complete overlap among sampling methods), whereas an IS value of 0 indicates that the two treatments have no common species (there is no similarity in community composition between sampling methods).

2.5. Statistical Data Analysis. All variables were tested for normality and the strongly skewed variables were transformed prior to analyses if necessary to meet the assumption of normality and homogeneity of variances. Variables expressed as percentages (\%) were arcsine-squareroot $(+0.5)$ transformed and number of species or counts of individuals were $\ln (x+1)$ transformed. However, when $\log$ transformation of the raw data was performed and if the data was still skewed, an appropriate nonparametric test was applied. Back-transformed data are reported.

The differences in butterfly individuals and species, evenness and diversity between the 26 study sites were tested with General Linear Model (GLM) analysis of variance (ANOVA) in Minitab release version 15. Where the GLM test indicated significant differences, post-hoc Fishers' least significant difference (LSD) test was used.

To find out whether large-scale fields supported rich and diverse butterfly communities compared to small-scale fields, the General Linear Model (GLM) was applied with Fisher's protected least significant difference (LSD) test for mean separation at $5 \%$ probability.

Data on butterfly attraction to different habitat/landuse types found in the 26 study sites was skewed even after transformation. Therefore, to identify best habitat/land-use types for butterflies inhabiting farmlands, a nonparametric test was applied to investigate the effect of habitat types on the richness and abundance of butterflies.

The relationships of foraging butterfly species and "environmental factors" were explored using CANOCO program, version 4.5 [29]. A unimodal direct gradient analysis of partial canonical correspondence analysis (CCA) was used to relate the variation of butterfly communities to environmental variables. Different land-uses and seminatural habitats recorded on transects were put in the data matrix of independent environmental factors, whereas recorded foraging butterfly species were grouped in the data matrix of dependent variables. The ordination analysis was based on all butterfly species that were entered in the model with 10 environmental factors. Environmental variables were selected using manual forward selection. Under a 
reduced model for the canonical axes (499 randomizations), Monte Carlo permutation tests were used to assess statistical significance of the association (linear relationship) between butterfly community species composition and environmental factors. Results from the CCA produce eigenvalues that are used to describe how much variance is explained by each ordination axis, thus measuring the importance of each axis. Whenever the significance of the whole ordination was obtained, a forward stepwise method was used to determine which environmental variables were correlated to axes and explained variation in butterfly species composition. A test for significance of correlation of environmental factors with the 4 different axes was conducted using Spearman rank of correlation. To illustrate the relationships between environmental variables and species, biplots of the respective partial CCA were produced.

\section{Results}

3.1. Butterfly Assemblages. Overall 56315 butterfly individuals belonging 6 families, 15 subfamilies, 24 tribes, 95 genera, and 331 species (Table 2) were sampled across the 26 study sites. There were 120 singleton species, 80 doubleton species, and 59 rare species. The highest estimated species richness was captured by hand nets (where Jacknife-1 revealed a very steep final slope). The species accumulation curve was relatively steep, not reaching an asymptote in hand net and transect counts although beginning to reach a plateau by round four in banana-bait trapping method. Similarly, the overall species accumulation curves for butterflies did not saturate. Because the curve did not saturate, this indicated that not all species of butterfly present in central Uganda were sampled. Overall, the total number of observed species was 331 while Jacknife1 predicted a total of 360 species. This indicates that $91.2 \%$ (331 of 360 estimated species) of the species present in the study area during the period of study were sampled.

In this study, the highest species richness was captured in hand nets (Figure 3). Most (75\% of total individuals) butterflies were recorded through line transect counts and very relatively individuals were captured by hand net (19\%) and banana-bait trap (6\%). Hence, hand-net and banana-baits methods sampled statistically $(P>0.05)$ similar butterfly individuals.

There were differences between average similarity index values (Sorensen similarity index) of species shared between the three sampling methods. The percentage of shared species (measured here as \% of similarity in species composition) between the sampling methods was highest between hand net and transects counts (41.9\%) followed by hand net and banana-bait traps (31.8\%) and least between bananabaits and transect counts $(21.5 \%)$. These results also indicate that use of multiple techniques can considerably increase the number of species.

The abundance of different butterfly taxa varied among sampling methods. Overall, the most abundant species was Bicyclus safitza (14.5\%) followed by Acraea acerata (6.3\%), Catopsilia florella (6.5\%), and Junonia sophia (6.1\%). Also, the most abundant genus was Bicyclus (21.6\%) followed by Acraea (13\%), Junonia (6.1\%), and Eurema (5.7\%). For the fruit bait traps, Bicyclus safitza (23.3\%), followed by Eurytela drope (6.4\%), were the most abundant species. The most abundant genus trapped using banana-bait trap was Bicyclus (45.2\%) followed by Eurytela (8.3\%), Acraea (5.9\%), Junonia (5.7\%), and Neptidopsis (4.5\%).

Most (44\%) of the 331 species sampled were "forestdependent species," followed by widespread species (31.4\%) and open habitat specialists (13\%) (Table 2). Species belonging to the other ecological trait categories constituted less than $12 \%$ of the total species sampled. Forest edge/woodland had the least proportion of species sampled (Table 2).

Variation in butterfly abundance, species richness, and diversity between the 26 study sites within the different sampling methods were striking (Table 3 ). The highest abundance was associated with Kiweebwa study site whereas the site with lowest abundance was Nonve (Table 3). The highest butterfly species richness was found in Lukalu and the lowest in Nonve study sites (Table 3). The highest diversity index value was found in Kimuli study site and the lowest in Nonve study site (Table 3). There was no clear pattern for the land-use intensity gradient. Study sites located in regions with low land-use intensity were not necessarily species study sites that supported high species richness. For example, the highest specie richness was recorded in Lukalu site that is located in region with medium land-use intensity. However, study sites with very high land use intensity were the least species-rich sites: Nonve and Luwunga (Table 3).

The pattern of butterfly species richness and abundance varied across sampling rounds and sampling methods. Average butterfly species richness ranged from 19 to 32 species across sampling rounds, with the highest richness occurring during the first round and the least in the fifth round of data collection. Butterfly abundance was higher in the first and second sampling rounds than in the rest of the rounds. Overall, butterfly species richness and abundance varied seasonally, with more species and higher densities in the rainy seasons (wet months) compared with the dry season (dry months).

3.2. Environmental Factors Determining Occurrence of Different Species. Butterfly community was significantly related to environmental factors measured (Figure 4; Table 4: CCA; sum of all canonical variables $=1.635 ; F=1.286, P=$ 0.01603 for all canonical axes). The first, second, third, and fourth axes explain $26.6 \%, 24.2 \%, 23.2 \%$, and $20.4 \%$ of the variance, respectively, in the model. The correlation of the environmental variables with the axes is summarized in Table 5. Few environmental factors significantly correlated with axes (Table 5). Axis 3 had a high loading for the most determinant factors of butterflies in farmland of central Uganda: small-scale agroforestry fields with fruit tree species, large coffee plantations in the margins of native forest fragments, swampy habitats, reclaimed wetlands, stream-edges, Eucalyptus/Pine plantations, woodlands, grasslands, large tea plantations in vicinity of wetlands/swamps, small-scale monoculture/polyculture fields of annual/biannual crops, 
TABle 2: Systematic list of butterfly species collected from farmlands of central Uganda in 2006. The species are arranged per family, subfamily, and par ecological category.

\begin{tabular}{|c|c|c|c|}
\hline Family & Subfamily & Species & E-cat \\
\hline Riodinidae & Nemobiinae & Abisara neavei neavei (Riley) & FDS \\
\hline Hesperiidae & Hesperiinae & Acleros mackenii olaus (Plötz) & FEW \\
\hline Nymphalidae & Heliconiinae & Acraea lycoa (Godart) & $\mathrm{N}$ \\
\hline Nymphalidae & Heliconiinae & Acraea acerata (Hewitson) & WSS \\
\hline Nymphalidae & Heliconiinae & Acraea acrita (Hewitson) & $\mathrm{N}$ \\
\hline Nymphalidae & Heliconiinae & Acraea aganice (Hewitson) & SWSS \\
\hline Nymphalidae & Heliconiinae & Acraea alicia (Sharpe) & $\mathrm{N}$ \\
\hline Nymphalidae & Heliconiinae & Acraea asboloplintha (Karsch) & SWSS \\
\hline Nymphalidae & Heliconiinae & Acraea baxteri philos & FEW \\
\hline Nymphalidae & Heliconiinae & Acraea bonasia bonasia & FDS \\
\hline Nymphalidae & Heliconiinae & Acraea boopis ama (Pierre) & FDS \\
\hline Nymphalidae & Heliconiinae & Acraea cabira (Hopffer) & FEW \\
\hline Nymphalidae & Heliconiinae & Acraea egina egina (Cramer) & WSS \\
\hline Nymphalidae & Heliconiinae & Acraea encedon encedon (Linnaeus) & WSS \\
\hline Nymphalidae & Heliconiinae & Acraea eponina eponina (Cramer) & WSS \\
\hline Nymphalidae & Heliconiinae & Acraea jodutta jodutta (Fabricius) & FDS \\
\hline Nymphalidae & Heliconiinae & Acraea johnstoni johnstoni (Godman) & FEW \\
\hline Nymphalidae & Heliconiinae & Acraea leucographa (Ribbe) & FDS \\
\hline Nymphalidae & Heliconiinae & Acraea macarista macarista (Sharpe) & FDS \\
\hline Nymphalidae & Heliconiinae & Acraea natalica natalica (Boisduval) & $\mathrm{N}$ \\
\hline Nymphalidae & Heliconiinae & Acraea neobule (Doubleday) & WSS \\
\hline Nymphalidae & Heliconiinae & Acraea penelope vitrea (Eltringham) & FDS \\
\hline Nymphalidae & Heliconiinae & Acraea perenna perenna (Doubleday) & FEW \\
\hline Nymphalidae & Heliconiinae & Acraea pharsalus pharsalus (Ward) & $\mathrm{N}$ \\
\hline Nymphalidae & Heliconiinae & Acraea poggei nelson (Grose-Smith \& Kirby) & FDS \\
\hline Nymphalidae & Heliconiinae & Acraea pudorella pudorella (Aurivillius) & $\mathrm{N}$ \\
\hline Nymphalidae & Heliconiinae & Acraea quirinalis (Grose-Smith) & FDS \\
\hline Nymphalidae & Heliconiinae & Acraea semivitrea (Aurivillius) & FDS \\
\hline Nymphalidae & Heliconiinae & Acraea servona (Godart) & FDS \\
\hline Nymphalidae & Heliconiinae & Acraea sotikensis (Sharpe) & FDS \\
\hline Nymphalidae & Heliconiinae & Acraea uvui uvui (Grose-Smith) & FEW \\
\hline Nymphalidae & Heliconiinae & Acraea ventura ochrascens (Hewitson) & SWSS \\
\hline Nymphalidae & Heliconiinae & Acraea viviana (Staudinger) & FEW \\
\hline Nymphalidae & Heliconiinae & Acraea zetes zetes (Linnaeus) & WSS \\
\hline Nymphalidae & Heliconiinae & Acraea zonata (Hewitson) & $\mathrm{N}$ \\
\hline Lycaenidae & Polyommatinae & Actizera lucida lucida (Trimen) & WSS \\
\hline Lycaenidae & Polyommatinae & Actizera stellata (Trimen) & OHPS \\
\hline Lycaenidae & Lipteninae & Alaena picata (Sharpe) & $\mathrm{N}$ \\
\hline Lycaenidae & Theclinae & Aloeides conradsi (Aurivillius) & $\mathrm{N}$ \\
\hline Nymphalidae & Danainae & Amauris albimaculata (Butler) & FDS \\
\hline Nymphalidae & Danainae & Amauris hecate hecate (Butler) & FDS \\
\hline Nymphalidae & Danainae & Amauris niavius niavius (Linnaeus) & WSS \\
\hline Nymphalidae & Danainae & Amauris tartarea (Mabille) & FEW \\
\hline Nymphalidae & Danainae & Amauris ochlea (Boisduval) & FEW \\
\hline Nymphalidae & Charaxinae & Antanartia delius delius (Drury) & FDS \\
\hline Lycaenidae & Polyommatinae & Anthene amarah amrah (Guérin-Ménéeville) & OHPS \\
\hline Lycaenidae & Polyommatinae & Anthene butleri (Oberthür) & WSS \\
\hline Lycaenidae & Polyommatinae & Anthene kampala & FEW \\
\hline Lycaenidae & Polyommatinae & Anthene larydas (Cramer) & FEW \\
\hline Lycaenidae & Polyommatinae & Anthene lunulata (Trimen) & FEW \\
\hline
\end{tabular}


Table 2: Continued.

\begin{tabular}{|c|c|c|c|}
\hline Family & Subfamily & Species & E-cat \\
\hline Lycaenidae & Polyommatinae & Anthene schoutedeni (Huelstaert) & FDS \\
\hline Pieridae & Pierinae & Appias sylvia ugandensis (Bernardi) & FDS \\
\hline Nymphalidae & Biblidinae & Ariadne albifascia (Joicey \& Talbot) & FDS \\
\hline Nymphalidae & Biblidinae & Ariadne enotrea suffusa (Joicey \& Talbot) & FEW \\
\hline Nymphalidae & Biblidinae & Ariadne pangenstecheri (Suffert) & FEW \\
\hline Hesperiidae & Hesperiinae & Artitropa milleri milleri (Holland) & WSS \\
\hline Lycaenidae & Theclinae & Axiocerses harpax ugandana (Clench) & $\mathrm{N}$ \\
\hline Lycaenidae & Polyommatinae & Azanus natalensis (Trimen) & MS \\
\hline Lycaenidae & Polyommatinae & Azanus ubaldus (Cramer) & MS \\
\hline Pieridae & Pierinae & Belenois creona (Cramer) & $\mathrm{N}$ \\
\hline Pieridae & Pierinae & Belenois raffrayi extendens (Joicey \& Talbot) & FDS \\
\hline Pieridae & Pierinae & Belenois solilucus (Butler) & FEW \\
\hline Pieridae & Pierinae & Belenois aurota (Fabricius) & MS \\
\hline Pieridae & Pierinae & Belenois creona severina (Stol) & MS \\
\hline Pieridae & Pierinae & Belenois subeida sylvander (Grose-Smith) & $\mathrm{N}$ \\
\hline Pieridae & Pierinae & Belenois theora theora (Doubleday) & FEW \\
\hline Pieridae & Pierinae & Belenois thysa (Hopffer) & FEW \\
\hline Nymphalidae & Satyrinae & Bicyclus buea (Strand) & FDS \\
\hline Nymphalidae & Satyrinae & Bicyclus funebris (Guérin-Ménéville) & FDS \\
\hline Nymphalidae & Satyrinae & Bicyclus mollitia & SWSS \\
\hline Nymphalidae & Satyrinae & Bicyclus safitza safitza (Hewitson) & WSS \\
\hline Nymphalidae & Satyrinae & Bicyclus abnormis (Dudgeon) & WSS \\
\hline Nymphalidae & Satyrinae & Bicyclus anynana anynana (Butler) & OHPS \\
\hline Nymphalidae & Satyrinae & Bicyclus campina ocelligera (Butler) & $\mathrm{N}$ \\
\hline Nymphalidae & Satyrinae & Bicyclus campinus (Aurivillius) & SWSS \\
\hline Nymphalidae & Satyrinae & Bicyclus campus (Karsch) & $\mathrm{N}$ \\
\hline Nymphalidae & Satyrinae & Bicyclus dentatus (Sharpe) & FEW \\
\hline Nymphalidae & Satyrinae & Bicyclus ena (Hewitson) & OHPS \\
\hline Nymphalidae & Satyrinae & Bicyclus evadne elionas (Hewitson) & $\mathrm{N}$ \\
\hline Nymphalidae & Satyrinae & Bicyclus golo (Aurivillius) & FDS \\
\hline Nymphalidae & Satyrinae & Bicyclus hewitsoni (Doumet) & FDS \\
\hline Nymphalidae & Satyrinae & Bicyclus istaris (Plötz) & FEW \\
\hline Nymphalidae & Satyrinae & Bicyclus jefferyi (Fox) & FEW \\
\hline Nymphalidae & Satyrinae & Bicyclus kenia (Rogernhofer) & FDS \\
\hline Nymphalidae & Satyrinae & Bicyclus mandanes (Hewitson) & FDS \\
\hline Nymphalidae & Satyrinae & Bicyclus mollitia (Karsch) & $\mathrm{N}$ \\
\hline Nymphalidae & Satyrinae & Bicyclus sandace (Hewitson) & FDS \\
\hline Nymphalidae & Satyrinae & Bicyclus saussurei saussurei (Condamin) & FDS \\
\hline Nymphalidae & Satyrinae & Bicyclus smithi smithi (Aurivillius) & FDS \\
\hline Nymphalidae & Satyrinae & Bicyclus sophrosyne sophrosyne (Plötz) & SWSS \\
\hline Nymphalidae & Satyrinae & Bicyclus sp. 1 & $\mathrm{~N}$ \\
\hline Nymphalidae & Satyrinae & Bicyclus sp.2 & $\mathrm{N}$ \\
\hline Nymphalidae & Satyrinae & Bicyclus vulgaris (Butler) & WSS \\
\hline Hesperiidae & Hesperiinae & Borbo fatuellus fatuellus (Hopffer) & WSS \\
\hline Hesperiidae & Hesperiinae & Borbo holtzii (Plötz) & OHPS \\
\hline Hesperiidae & Hesperiinae & Borbo kaka (Evans) & FDS \\
\hline Nymphalidae & Biblidinae & Byblia anvatara acheloia (Wallengren) & MS \\
\hline Nymphalidae & Biblidinae & Byblia ilithyia (Drury) & $\mathrm{N}$ \\
\hline Lycaenidae & Polyommatinae & Cacyreus palemon palemon (Stoll) & OHPS \\
\hline Lycaenidae & Hesperiinae & Caenides luehderi laura (Evans) & $\mathrm{N}$ \\
\hline Pieridae & Coliadinae & Catopsilia florella (Fabricius) & MS \\
\hline
\end{tabular}


Table 2: Continued.

\begin{tabular}{|c|c|c|c|}
\hline Family & Subfamily & Species & E-cat \\
\hline Hesperiidae & Pyrginae & Celaenorrhinus galenus (Fabricius) & FDS \\
\hline Hesperiidae & Pyrginae & Celaenorrhinus ovalis (Evans) & $\mathrm{N}$ \\
\hline Hesperiidae & Pyrginae & Celaenorrhinus proxima proxima (Mabille) & FDS \\
\hline Nymphalidae & Charaxinae & Charaxes acuminatus (Thurau) & FDS \\
\hline Nymphalidae & Charaxinae & Charaxes anticlea suna (van Someren) & FEW \\
\hline Nymphalidae & Charaxinae & Charaxes aubyni ecketti & $\mathrm{N}$ \\
\hline Nymphalidae & Charaxinae & Charaxes bipunctatus ugandensis (van Someren) & FDS \\
\hline Nymphalidae & Charaxinae & Charaxes brutus (Cramer) & FEW \\
\hline Nymphalidae & Charaxinae & Charaxes chepalungu (van Someren) & $\mathrm{N}$ \\
\hline Nymphalidae & Charaxinae & Charaxes contrarius (Weymer) & $\mathrm{N}$ \\
\hline Nymphalidae & Charaxinae & Charaxes dilutusngonga & FDS \\
\hline Nymphalidae & Charaxinae & Charaxes etesipe (Rothschild) & FEW \\
\hline Nymphalidae & Charaxinae & Charaxes ethalion nyanzae (de Boisduval) & WSS \\
\hline Nymphalidae & Charaxinae & Charaxes etheocles (van Someren \& Jackson) & FDS \\
\hline Nymphalidae & Charaxinae & Charaxes etheocles etheocles (Cramer) & FDS \\
\hline Nymphalidae & Charaxinae & Charaxes eupale latimargo (Joicey \& Talbot) & $\mathrm{N}$ \\
\hline Nymphalidae & Charaxinae & Charaxes guderiana rabaiensis (Poulton) & $\mathrm{N}$ \\
\hline Nymphalidae & Charaxinae & Charaxes hildebrandti hildebrandti (Dewitz) & FDS \\
\hline Nymphalidae & Charaxinae & Charaxes lactetinctus lactetinctus (Karsch) & OHPS \\
\hline Nymphalidae & Charaxinae & Charaxes lucretius maximus (van Someren) & FDS \\
\hline Nymphalidae & Charaxinae & Charaxes nobilis claudei (le Moult) & FDS \\
\hline Nymphalidae & Charaxinae & Charaxes numenes numenes (Hewitson) & FEW \\
\hline Nymphalidae & Charaxinae & Charaxes plantroui (Minig) & $\mathrm{N}$ \\
\hline Nymphalidae & Charaxinae & Charaxes pleione (Godart) & $\mathrm{N}$ \\
\hline Nymphalidae & Charaxinae & Charaxes protoclea azota (Hewitson) & FEW \\
\hline Nymphalidae & Charaxinae & Charaxes smaragdalis butleri (Rothschild) & FDS \\
\hline Nymphalidae & Charaxinae & Charaxes tiridates tiridates (Cramer) & FDS \\
\hline Nymphalidae & Charaxinae & Charaxes varanes vologeses (Mabille) & WSS \\
\hline Nymphalidae & Charaxinae & Charaxes viola picta (van Someren \& Jackson) & OHPS \\
\hline Nymphalidae & Charaxinae & Charaxes xiphares & FDS \\
\hline Nymphalidae & Charaxinae & Charaxes zoolina zoolina (Westwood) & OHPS \\
\hline Lycaenidae & Polyommatinae & Chilades kedonga (Grose-Smith) & OHPS \\
\hline Hesperiidae & Hesperiinae & Chondrolepsis niveicornis niveicornis (Plötz) & FDS \\
\hline Lycaenidae & Lipteninae & Cocyreus lingens & $\mathrm{N}$ \\
\hline Hesperiidae & Coeliadinae & Coeliades anchises anchises (Gerstäcker) & MS \\
\hline Hesperiidae & Coeliadinae & Coeliades chalybe (Westwood) & FDS \\
\hline Hesperiidae & Coeliadinae & Coeliades forestan forestan (Stoll) & WSS \\
\hline Hesperiidae & Coeliadinae & Coeliades hanno & FDS \\
\hline Nymphalidae & Satyrinae & Coenyropsis carcassoni (Kielland) & OHPS \\
\hline Pieridae & Coliadinae & Colias electo pseudohecate (Berger) & MS \\
\hline Pieridae & Pierinae & Colotis antevippe zera (Lucas) & OHPS \\
\hline Pieridae & Pierinae & Colotis aurigineus (Butler) & WSS \\
\hline Pieridae & Pierinae & Colotis chrysonome (Klug) & OHPS \\
\hline Pieridae & Pierinae & Colotis danae (Fabricius) & WSS \\
\hline Pieridae & Pierinae & Colotis euippe (Linnaeus) & WSS \\
\hline Pieridae & Pierinae & Colotis venosus (Staudinger) & OHPS \\
\hline Lycaenidae & Polyommatinae & Cupidopsis cissus (Godart) & WSS \\
\hline Lycaenidae & Polyommatinae & Cupidopsis jobates jobates (Hopffer) & WSS \\
\hline Nymphalidae & Limenitidinae & Cymothoe elgesta confusa (Aurivillius) & $\mathrm{N}$ \\
\hline Nymphalidae & Nymphalinae & Cymothoe lurida butleri (Grünberg) & FDS \\
\hline Nymphalidae & Nymphalinae & Cymothoe althea althea & $\mathrm{N}$ \\
\hline
\end{tabular}


Table 2: Continued.

\begin{tabular}{|c|c|c|c|}
\hline Family & Subfamily & Species & E-cat \\
\hline Nymphalidae & Limenitidinae & Cymothoe herminia johnstoni (Butler) & FDS \\
\hline Nymphalidae & Limenitidinae & Cymothoe hobarti hobarti (Butler) & FDS \\
\hline Nymphalidae & Limenitidinae & Cymothoe weymeri weymeri (Suffert) & $\mathrm{N}$ \\
\hline Nymphalidae & Danainae & Danaus chrysippus chrysippus (Linnaeus) & MS \\
\hline Nymphalidae & Danainae & Danaus dorripus (Klug) & MS \\
\hline Pieridae & Pierinae & Dixeia orbona vidua (Butler) & WSS \\
\hline Pieridae & Pierinae & Dixeia pigea (Boisduval) & WSS \\
\hline Pieridae & Liphyrinae & Dixeia spilleri spilleri (Spiller) & $\mathrm{N}$ \\
\hline Lycaenidae & Polyommatinae & Eicochrysops messapus (Bethune\&Baker) & $\mathrm{N}$ \\
\hline Lycaenidae & Polyommatinae & Eicochrysops Hippocrates (Fabricius) & $\mathrm{N}$ \\
\hline Lycaenidae & Polyommatinae & Eicochrysops masai (Bethune-Baker) & OHPS \\
\hline Hesperiidae & Pyrginae & Eretis lugens (Rogenhofer) & WSS \\
\hline Pieridae & Coliadinae & Eronia cleodora (Hubne) & OHPS \\
\hline Lycaenidae & Polyommatinae & Euchrysops nilotica (Aurivillius) & $\mathrm{N}$ \\
\hline Lycaenidae & Polyommatinae & Euchrysops malathana (Boisduval) & OHPS \\
\hline Lycaenidae & Polyommatinae & Euchrysops osiris (Hopffer) & WSS \\
\hline Lycaenidae & Polyommatinae & Euchrysops subpallida (Bethune-Baker) & $\mathrm{N}$ \\
\hline Nymphalidae & Nymphalinae & Euphaedra preussi preussi (Staudinger) & $\mathrm{N}$ \\
\hline Pieridae & Coliadinae & Eurema brigitta brigitta (Stoll) & MS \\
\hline Pieridae & Coliadinae & Eurema desjardinsi oberthuri (Mabille) & MS \\
\hline Pieridae & Coliadinae & Eurema floricola orientis (Butler) & FDS \\
\hline Pieridae & Coliadinae & Eurema hecabe solifera (Butler) & MS \\
\hline Pieridae & Coliadinae & Eurema regularis regularis (Butler) & WSS \\
\hline Pieridae & Coliadinae & Eurema senegalensis (Boisduval) & FDS \\
\hline Nymphalidae & Nymphalinae & Eurytela dryope angulate (Aurivillius) & WSS \\
\hline Nymphalidae & Nymphalinae & Eurytela alinda (Mabille) & $\mathrm{N}$ \\
\hline Nymphalidae & Nymphalinae & Eurytela hiarbas lita (Rothschild \& Jordan) & FEW \\
\hline Hesperiidae & Hesperiinae & Freyeria trochylus (Freyer) & WSS \\
\hline Hesperiidae & Hesperiinae & Gegenes hottentota (Latreille) & OHPS \\
\hline Hesperiidae & Hesperiinae & Gegenes niso brevicornis (Plötz) & $\mathrm{N}$ \\
\hline Hesperiidae & Hesperiinae & Gegenes pumilio gambica (Mabille) & OHPS \\
\hline Nymphalidae & Satyrinae & Gnophodes betsimena diversa (Trimen) & FDS \\
\hline Papilionidae & Papilioninae & Graphium policenes (Cramer) & $\mathrm{N}$ \\
\hline Papilionidae & Papilioninae & Graphium antheus & FEW \\
\hline Papilionidae & Papilioninae & Graphium leonidas Leonidas (Fabricius) & MS \\
\hline Papilionidae & Papilioninae & Graphium polistratus (Grose-smith) & FEW \\
\hline Nymphalidae & Satyrinae & Hallelesis asochis & $\mathrm{N}$ \\
\hline Nymphalidae & Satyrinae & Hallelesis halyma & $\mathrm{N}$ \\
\hline Nymphalidae & Satyrinae & Halocerina angulata & $\mathrm{N}$ \\
\hline Nymphalidae & Nymphalinae & Hamanumida daedalus (Fabricius) & WSS \\
\hline Nymphalidae & Satyrinae & Henotesia peitho (Plötz) & WSS \\
\hline Nymphalidae & Satyrinae & Henotesia perpicua (Trimen) & OHPS \\
\hline Nymphalidae & Nymphalinae & Hypokooelates ugandae (Talbot) & $\mathrm{N}$ \\
\hline Nymphalidae & Nymphalinae & Hypolimnas misippus (Linnaeus) & MS \\
\hline Nymphalidae & Nymphalinae & Hypolimnas salmacis magnifica (Rothschild) & FDS \\
\hline Lycaenidae & Theclinae & Hypolycaena hatita ugandae (Sharpe) & FDS \\
\hline Lycaenidae & Theclinae & Iolaus poultoni (Riley) & FDS \\
\hline Nymphalidae & Heliconiinae & Issoria hanningtoni jeanneli (Bernardi) & FEW \\
\hline Nymphalidae & Nymphalinae & Junonia natalica (Felder) & FEW \\
\hline Nymphalidae & Nymphalinae & Junonia chorimene (Guérin-Ménéville) & OHPS \\
\hline Nymphalidae & Nymphalinae & Junonia eonone eonone (Linnaeus) & WSS \\
\hline
\end{tabular}


Table 2: Continued.

\begin{tabular}{|c|c|c|c|}
\hline Family & Subfamily & Species & E-cat \\
\hline Nymphalidae & Nymphalinae & Junonia hierta cebrene (Trimen) & MS \\
\hline Nymphalidae & Nymphalinae & Junonia orithya madagascariensis (Guenee) & $\mathrm{N}$ \\
\hline Nymphalidae & Nymphalinae & Junonia sophia infracta (Butler) & $\mathrm{N}$ \\
\hline Nymphalidae & Nymphalinae & Junonia stygia gregorii (Butler) & FEW \\
\hline Nymphalidae & Nymphalinae & Junonia terea elgiva (Drury) & WSS \\
\hline Nymphalidae & Nymphalinae & Junonia westermannii suffusa (Rothschild \& Jordan) & FDS \\
\hline Hesperiidae & Hesperinae & Kedestes rogersi $($ Druce $)$ & OHPS \\
\hline Lycaenidae & Polyommatinae & Lepidochrysops desmondi (Stempffer) & $\mathrm{N}$ \\
\hline Lycaenidae & Polyommatinae & Lepidochrysops elgonae (Stempffer) & $\mathrm{N}$ \\
\hline Lycaenidae & Polyommatinae & Lepidochrysops cf jansei (van Someren) & WSS \\
\hline Lycaenidae & Polyommatinae & Lepidochrysops kitale (Stempffer) & $\mathrm{N}$ \\
\hline Lycaenidae & Polyommatinae & Lepidochrysops parsimon parsimon (Fabricius) & OHPS \\
\hline Pieridae & Pierinae & Leptosia alcesta inalcesta (Bernardi) & WSS \\
\hline Pieridae & Pierinae & Leptosia nupta nupta (Butler) & FDS \\
\hline Pieridae & Pierinae & Leptosia wigginsi (Dixey) & FDS \\
\hline Nymphalidae & Libytheinae & Libythea labdaca (Westwood) & MS \\
\hline Lycaenidae & Lipteninae & Liptena xanthostola xantha (Grose-Smith) & $\mathrm{N}$ \\
\hline Nymphalidae & Nymphalinae & Mallika jacksoni (Sharpe) & OHPS \\
\hline Nymphalidae & Satyrinae & Melanitis leda (Linnaeus) & WSS \\
\hline Lycaenidae & Lipteninae & Mimacraeakrausei & $\mathrm{N}$ \\
\hline Lycaenidae & Lipteninae & Mimacraea marshalli (Trimen) & FEW \\
\hline Hesperiidae & Hesperiinae & Monza alberti (Holland) & FDS \\
\hline Hesperiidae & Hesperiinae & Monza cretacea (Snellen) & WSS \\
\hline Pieridae & Pierinae & Mylothris aburi (Collins \& Larsen) & $\mathrm{N}$ \\
\hline Pieridae & Pierinae & Mylothris agathina (Cramer) & WSS \\
\hline Pieridae & Pierinae & Mylothris chloris clarissa (Butler) & WSS \\
\hline Pieridae & Pierinae & Mylothris continua continua (Aurivillius) & FDS \\
\hline Pieridae & Pierinae & Mylothris hilara (Karsch) & FDS \\
\hline Pieridae & Pierinae & Mylothris ochracea (Aurivillius) & $\mathrm{N}$ \\
\hline Pieridae & Pierinae & Mylothris sjoestedti sjoestedti (Aurivillius) & FDS \\
\hline Lycaenidae & Theclinae & Myrina dermaptera nyasae (Talbot) & OHPS \\
\hline Pieridae & Pierinae & Nepheronia argia (Fabricius) & FDS \\
\hline Pieridae & Pierinae & Nepheronia pharis silvanus (Stoneham) & FDS \\
\hline Pieridae & Pierinae & Nepheronia thalassina (de Boisduval) & FEW \\
\hline Nymphalidae & Nymphalinae & Neptidopsis fulgurate (Rothschild \& Jordan) & $\mathrm{N}$ \\
\hline Nymphalidae & Nymphalinae & Neptidopsis ophione nucleate (Mabille) & FEW \\
\hline Nymphalidae & Limenitidinae & Neptis alta (Overlaet) & FDS \\
\hline Nymphalidae & Limenitidinae & Neptis carcassoni (van Son) & $\mathrm{N}$ \\
\hline Nymphalidae & Limenitidinae & Neptis clarei (Aurivillius) & FDS \\
\hline Nymphalidae & Limenitidinae & Neptis jordani (Neave) & $\mathrm{N}$ \\
\hline Nymphalidae & Limenitidinae & Neptis katama (Collins\&Larsen) & $\mathrm{N}$ \\
\hline Nymphalidae & Limenitidinae & Neptis kiriakoffi (Overlaet) & WSS \\
\hline Nymphalidae & Limenitidinae & Neptis laeta (Overlaet) & WSS \\
\hline Nymphalidae & Limenitidinae & Neptis melicerta melicerta (Hewitson) & FDS \\
\hline Nymphalidae & Limenitidinae & Neptis metella metella (Doubleday) & FEW \\
\hline Nymphalidae & Limenitidinae & Neptis morosa (Overlaet) & WSS \\
\hline Nymphalidae & Limenitidinae & Neptis nemetes nemetes (Hewitson) & FEW \\
\hline Nymphalidae & Limenitidinae & Neptis occidentalis occidentalis (Rothschild) & FDS \\
\hline Nymphalidae & Limenitidinae & Neptis ochracea milbraedi (Gaede) & FDS \\
\hline Nymphalidae & Limenitidinae & Neptis penningtoni & $\mathrm{N}$ \\
\hline Nymphalidae & Limenitidinae & Neptis poultoni (Eltringham) & FDS \\
\hline
\end{tabular}


Table 2: Continued.

\begin{tabular}{|c|c|c|c|}
\hline Family & Subfamily & Species & E-cat \\
\hline Nymphalidae & Limenitidinae & Neptis saclava marpessa (Hopffer) & WSS \\
\hline Nymphalidae & Limenitidinae & Neptis strigata (Aurivillius) & FDS \\
\hline Nymphalidae & Limenitidinae & Neptis woodwardi woodwardi (Sharpe) & FDS \\
\hline Lycaenidae & Polyommatinae & Oboronia punctatus & $\mathrm{N}$ \\
\hline Lycaenidae & Lipteninae & Ornipholidotos peucetia peuceda (Grose-Smith) & FDS \\
\hline Papilionidae & Papilioninae & Papilio bromius (Suffert) & FEW \\
\hline Papilionidae & Papilioninae & Papilio dardanus polytropus (Brown) & WSS \\
\hline Papilionidae & Papilioninae & Papilio demodocus (Esper) & MS \\
\hline Papilionidae & Papilioninae & Papilio lormieri & FDS \\
\hline Papilionidae & Papilioninae & Papilio nireus lyaeus (Doubleday) & FEW \\
\hline Papilionidae & Papilioninae & Papilio nobilis (Rogenhofer) & FDS \\
\hline Papilionidae & Papilioninae & Papilio ophidicephalus (Oberthür) & $\mathrm{N}$ \\
\hline Papilionidae & Papilioninae & Papilio phorcas (Cramer) & FDS \\
\hline Papilionidae & Papilioninae & Papilio zoroastres joiceyi (Gabriel) & FDS \\
\hline Papilionidae & Hesperinae & Paracleros biguttulus (Mabille) & FEW \\
\hline Hesperiidae & Hesperinae & Pardaleodes incerta (Snellen) & FDS \\
\hline Hesperiidae & Hesperinae & Pardaleodes sator pusiella (Mabille) & FDS \\
\hline Nymphalidae & Heliconiinae & Pardopsis punctatissima (Boisduval) & WSS \\
\hline Lycaenidae & Lipteniinae & Pentila tachyroides tachyroides (Dewitz) & $\mathrm{N}$ \\
\hline Lycaenidae & Lipteniinae & Pentila pauli clarensis (Neave) & $\mathrm{N}$ \\
\hline Nymphalidae & Heliconiinae & Phalanta phalantha (Rothschild \& Jordan) & MS \\
\hline Pieridae & Pierinae & Pinacopteryx eriphia melanarge (Butler) & OHPS \\
\hline Pieridae & Pierinae & Pontia helice johnstoni (Crowley) & MS \\
\hline Nymphalidae & Nymphalinae & Precis antilope (Feishamel) & OHPS \\
\hline Nymphalidae & Nymphalinae & Precis archesia (Cramer) & OHPS \\
\hline Nymphalidae & Nymphalinae & Precis ceryne ceryne (Boiduval) & SWSS \\
\hline Nymphalidae & Nymphalinae & Precis coelestina & FEW \\
\hline Nymphalidae & Nymphalinae & Precis octavia sesamus (Trimen) & WSS \\
\hline Nymphalidae & Nymphalinae & Precis tugela (Trimen) & FEW \\
\hline Hesperiidae & Hesperinae & Prosopalpus debilis (Evans) & $\mathrm{N}$ \\
\hline Hesperiidae & Hesperinae & Protogoniomorpha temora & $\mathrm{N}$ \\
\hline Nymphalidae & Nymphalinae & Pseudacraea eurytus eurytus (Linnaeus) & FDS \\
\hline Nymphalidae & Nymphalinae & Pseudacraea lucretia protracta (Cramer) & FEW \\
\hline Nymphalidae & Nymphalinae & Pseudacraea boisduvali (Doubleday) & $\mathrm{N}$ \\
\hline Nymphalidae & Nymphalinae & Pseudargynnis hegemone (Godart) & FEW \\
\hline Nymphalidae & Nymphalinae & Pseudacraea lucretia expansa (Butler) & $\mathrm{N}$ \\
\hline Nymphalidae & Nymphalinae & Salamis cacta cacta fbricius & FDS \\
\hline Nymphalidae & Nymphalinae & Salamis parhassus (Drury) & FEW \\
\hline Nymphalidae & Nymphalinae & Sallya boisduvali (Wallengren) & MS \\
\hline Nymphalidae & Nymphalinae & Sallya garega garega (Karsch) & MS \\
\hline Nymphalidae & Nymphalinae & Sallya natalensis (Boisduval) & MS \\
\hline Nymphalidae & Nymphalinae & Sallya occidentalum occidentalium (Mabille) & MS \\
\hline Nymphalidae & Nymphalinae & Sallya umbrina (Karsch) & MS \\
\hline Hesperiidae & & Sarangesa laelius (Mabille) & WSS \\
\hline Lycaenidae & Pyrginae & Liptena maculata (Mabille) & OHPS \\
\hline Hesperiidae & Pyrginae & Septena xanthostola xantha & $\mathrm{N}$ \\
\hline Hesperiidae & Pyrginae & Spialia dromus (Plötz) & $\mathrm{N}$ \\
\hline Hesperiidae & Pyrginae & Spialia mafa higginsi (Evans) & $\mathrm{N}$ \\
\hline Hesperiidae & Pyrginae & Spialia mangana (Rebel) & $\mathrm{N}$ \\
\hline Hesperiidae & Pyrginae & Spialia ploetzi ploetzi (Aurivillius) & $\mathrm{N}$ \\
\hline Hesperiidae & Pyrginae & Spialia spio (Linnaeus) & OHPS \\
\hline
\end{tabular}


TABle 2: Continued.

\begin{tabular}{|c|c|c|c|}
\hline Family & Subfamily & Species & E-cat \\
\hline Hesperiidae & Hesperiinae & Tagiades flesus & $\mathrm{N}$ \\
\hline Lycaenidae & Lipteninae & Tetrarhanis diversa ilala (Riley) & $\mathrm{N}$ \\
\hline Nymphalidae & Danainae & Tirumala formosa morgeni (Honrath) & FEW \\
\hline Nymphalidae & Danainae & Tirumala petiverana (Doubleday \& Hewitson) & MS \\
\hline Lycaenidae & Polyommatinae & Triclema nigeriae (Aurivillius) & FEW \\
\hline Lycaenidae & Polyommatinae & Tuxentius margaritaceus margaritaceus (Sharpe) & WSS \\
\hline Lycaenidae & Polyommatinae & Uranothauma falkensteini (Dewitz) & $\mathrm{N}$ \\
\hline Nymphalidae & Nymphalinae & Vanessa cardui cardui (Linnaeus) & $\mathrm{N}$ \\
\hline Nymphalidae & Nymphalinae & Vanesula milca latifasciata (Joicey \& Talbot) & FEW \\
\hline Nymphalidae & Satyrinae & Ypthima albida albida (Butler) & FEW \\
\hline Nymphalidae & Satyrinae & Ypthima antennata antennata (van Son) & OHPS \\
\hline Nymphalidae & Satyrinae & Ypthima asterope asterope (Klug) & OHPS \\
\hline Nymphalidae & Satyrinae & Ypthima granulosa (Butler) & OHPS \\
\hline Nymphalidae & Satyrinae & Ypthima rhodesiana (Carcasson) & $\mathrm{N}$ \\
\hline Hesperiidae & Hesperiinae & Zezonia zeno (Trimen) & FEW \\
\hline Lycaenidae & Polyommatinae & Zizeeria knysna (Trimen) & WSS \\
\hline Lycaenidae & Polyommatinae & Zizina antanossa (Mabille) & WSS \\
\hline Lycaenidae & Polyommatinae & Zizula hylax (Fabricius) & WSS \\
\hline
\end{tabular}

Codes for different ecological habitat categories. E-cat: Ecological habitat category. FEW (f/m): "forest edge/woodland species"; FDS (F): "forest-dependent species"; MS (m): "migratory species"; OHPS (O): "open habitat specialist species"; SWSS (S): "swamp/wetland specialist species"; WSS (W): "widespread species" that are frequent fallows/grassland habitat users; N (U): "uncertain ecological category"—-species of unknown (un-described) habitat preference.

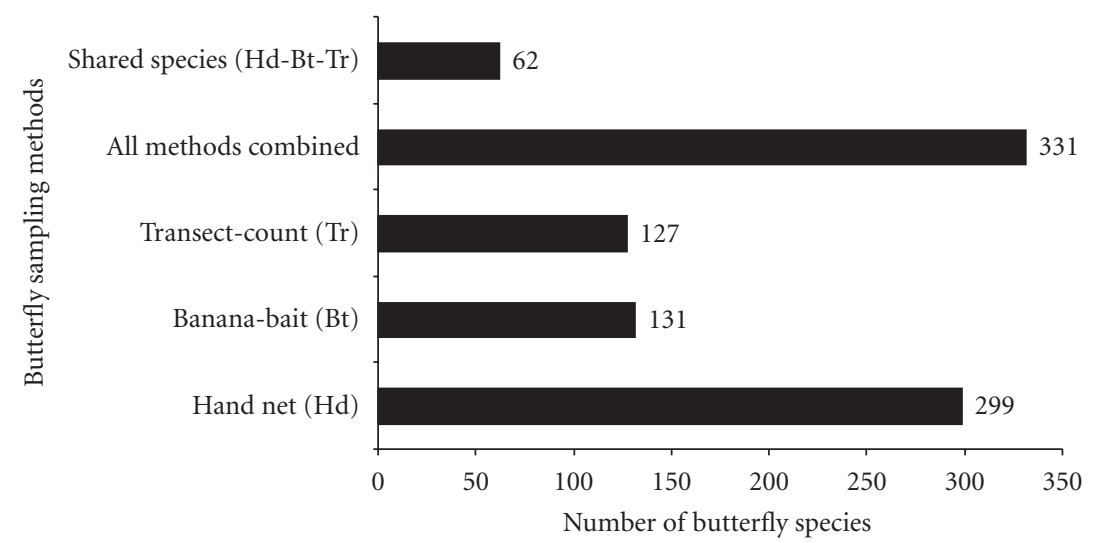

Figure 3: Number of butterfly species obtained using different sampling methods in farmlands of central Uganda during 2006.

small-scale grazing fields, hedgerows, and annual/biannual crop fields in the surrounding of forest reserves. Axis 2 was strongly significantly and positively related to environmental factors such as hedgerows, annual/biannual crop fields in the surrounding of forest reserves. Axis 1 was also negatively influenced by environmental factors such as large scale of tea plantations in vicinity of wetlands/swamps, but positively related to fallows. The fourth axis did not have high loadings but was observed to be frequently correlated (positively) to some other environmental factors: fallows and sugar plantation (Table 6). Environmental factors that were identified in the CCA as determinants for butterflies (Table 5) were also among the environmental factors observed to be significantly $(P<0.05$, LSD test $)$ associated with high species richness and abundance of butterflies (Table 6) in the coffeebanana agroforest systems. Across studies sites and sampling rounds, these habitats attracted a high number of butterflies both during the rainy and the dry seasons. Practically, few butterfly species were closely associated with "determinant environmental factors" previously identified in the CCA (Table 4) including swampy habitats, fallows, forest plantations, woodlands, stream-edges, tea plantations, and sugar plantations. For example, Belenois subeida, Bicyclus safitza, Melanitis leda, Charaxes varanes, and so forth appeared to be closely associated with fallows (Figure 4). Some of the species closely associated with swamp habitats and forest plantations may be considered as "farmland habitat specialists." The rest of species may be "generalist farmland habitats users." 
TABle 3: Butterfly species richness, abundance (individuals/2 ha/site), and diversity $\left(\mathrm{H}^{\prime}\right)$ in 26 different study sites in the coffee-banana farming system in central Uganda in 2006.

\begin{tabular}{|c|c|c|c|c|c|}
\hline Clusters & Sites & Land use intensity gradients & Species richness & Abundance & Diversity \\
\hline \multirow{4}{*}{ Bujjagali } & Bukose & High & $18.17 \pm 1.56 \mathrm{c}$ & $287.17 \pm 8.38 b$ & $2.31 \pm 0.28 \mathrm{~b}$ \\
\hline & Namizi-east & High & $23.41 \pm 3.06 b$ & $510.13 \pm 11.33 b$ & $2.24 \pm 0.17 \mathrm{e}$ \\
\hline & Namizi-west & High & $14.61 \pm 0.69 c$ & $230.23 \pm 8.11 b$ & $2.67 \pm 0.21 d$ \\
\hline & Nawangoma & High & $17.46 \pm 0.71 c$ & $274.33 \pm 6.09 b$ & $2.12 \pm 0.11 \mathrm{f}$ \\
\hline \multirow{4}{*}{ Kalagi } & Bamusuta & Medium & $20.54 \pm 0.63 c$ & $392.36 \pm 8.72 b$ & $2.95 \pm 0.21 \mathrm{c}$ \\
\hline & Kifu & Medium & $29.13 \pm 3.39 b$ & $541.57 \pm 12.03 b$ & $3.35 \pm 0.33 b$ \\
\hline & Kimwanyi & Medium & $31.81 \pm 3.59 b$ & $562.88 \pm 12.51 b$ & $3.36 \pm 0.37 b$ \\
\hline & Kiweebwa & Medium & $30.23 \pm 1.51 b$ & $1062.6 \pm 15.93 a$ & $3.24 \pm 0.25 b$ \\
\hline \multirow{2}{*}{ Kamuli } & Naikesa & High & $12.45 \pm 032 \mathrm{~d}$ & $151.87 \pm 3.37 \mathrm{c}$ & $1.81 \pm 0.22 \mathrm{~g}$ \\
\hline & Namulekya & High & $16.62 \pm 0.83 c$ & $213.34 \pm 7.74 b$ & $1.87 \pm 0.20 \mathrm{~g}$ \\
\hline \multirow{2}{*}{ Kaweri } & Luwunga & Very high & $18.37 \pm 0.51 c$ & $150.52 \pm 3.34 c$ & $1.69 \pm 0.21 \mathrm{~g}$ \\
\hline & Nonve & Very high & $11.76 \pm 0.58 \mathrm{~d}$ & $141.49 \pm 3.14 \mathrm{c}$ & $1.61 \pm 0.19 \mathrm{~g}$ \\
\hline \multirow{2}{*}{ Lugazi } & Kasaku & Very high & $26.22 \pm 2.31 b$ & $780.68 \pm 16.67 \mathrm{a}$ & $3.22 \pm 0.12 b$ \\
\hline & Sugar & Very high & $15.89 \pm 0.79 c$ & $269.57 \pm 8.99 b$ & $2.13 \pm 0.32 \mathrm{f}$ \\
\hline \multirow{2}{*}{ Mabira } & Bulyasi & Medium & $22.16 \pm 1.11 c$ & $409.58 \pm 9.10 b$ & $3.09 \pm 0.21 \mathrm{c}$ \\
\hline & Kinoni & Medium & $16.44 \pm 1.82 c$ & $316.12 \pm 7.02 b$ & $2.64 \pm 0.31 d$ \\
\hline \multirow{4}{*}{ Masaka } & Kasaala & Medium & $19.19 \pm 2.12 c$ & $421.48 \pm 9.37 b$ & $2.44 \pm 0.16 \mathrm{e}$ \\
\hline & Katwadde & Medium & $21.22 \pm 2.36 c$ & $426.59 \pm 9.48 b$ & $2.63 \pm 0.24 \mathrm{a}$ \\
\hline & Kiwaala & Medium & $28.87 \pm 3.23 b$ & $625.57 \pm 19.90 \mathrm{a}$ & $3.35 \pm 0.27 b$ \\
\hline & Mpugwe & Medium & $19.24 \pm 2.13 c$ & $217.69 \pm 7.840 b$ & $2.51 \pm 0.33 \mathrm{~d}$ \\
\hline \multirow{2}{*}{ Mpigi } & Lukalu & Medium & $44.65 \pm 4.95 a$ & $716.67 \pm 17.86 a$ & $3.41 \pm 0.25 \mathrm{a}$ \\
\hline & Mpanga & Medium & $38.19 \pm 4.23 a$ & $678.57 \pm 17.13 a$ & $3.56 \pm 0.26 a$ \\
\hline \multirow{4}{*}{ Nakaseke } & Kimuli & Low & $30.64 \pm 3.41 b$ & $918.27 \pm 21.71 \mathrm{a}$ & $3.71 \pm 0.27 \mathrm{a}$ \\
\hline & Kyetume & Low & $20.87 \pm 2.31 c$ & $368.67 \pm 8.87 b$ & $3.14 \pm 0.29 c$ \\
\hline & Lukumbi & Low & $13.67 \pm 2.51 \mathrm{c}$ & $282.88 \pm 7.44 b$ & $3.12 \pm 0.23 c$ \\
\hline & Segalye & Low & $27.65 \pm 3.06 b$ & $482.98 \pm 11.67 b$ & $3.01 \pm 0.23 c$ \\
\hline
\end{tabular}

Within columns, means followed by the same letter are not significantly different at $5 \%$ probability level (LSD test) test.

3.3. Effects of Farm Management Regimes on Butterflies. Although large monocultures (tea, sugar, coffee plantations) that are intensively managed appeared among important factors in the CCA analysis (Table 4), the species richness, abundance and diversity of butterflies were significantly $(P<$ 0.05 , LSD test) higher in traditional small-scale polyculture fields than in arge monoculture plantations (Table 7). These results indicated that small scale polyculture fields supported richer butterfly communities than did large monoculture (modernized fields).

\section{Discussion}

4.1. Determinants of Butterfly Species Distribution in CoffeeBanana Farming Systems. In this study, it is predicted that there would be no statistical differences among sites with different land uses, seminatural habitats, and farm management systems for the abundance, richness and diversity of butterflies since farmland habitats are subjected to constant anthropogenic disturbances. The results did not confirm the hypothesis since there were significant differences in species richness and abundance among the 26 study sites with different land-use types, habitat types and farm management regimes. Sites that hosted higher species richness and diversity were study sites that were riparian forest reserves or sites for which agricultural matrices were surrounded by forest patches and wetlands (see agroforests characteristics in Table 1). In contrast, the least diverse study sites or sites characterized by low butterfly species richness were also study sites of low habitat heterogeneity. These were also sites dominated by high management intensities (sites subjected to high cropping intensity) or sites located in regions with high land-use intensity gradients.

Overall butterfly species richness and density varied seasonally, with more species and higher densities in the rainy seasons (wet months) compared with the dry season (dry months). It is likely that seasonal changes in species 
TABLE 4: Summary of canonical correspondence analysis (CCA) ordination results relating butterfly community to environmental variables measured across 26 study sites in 2006.

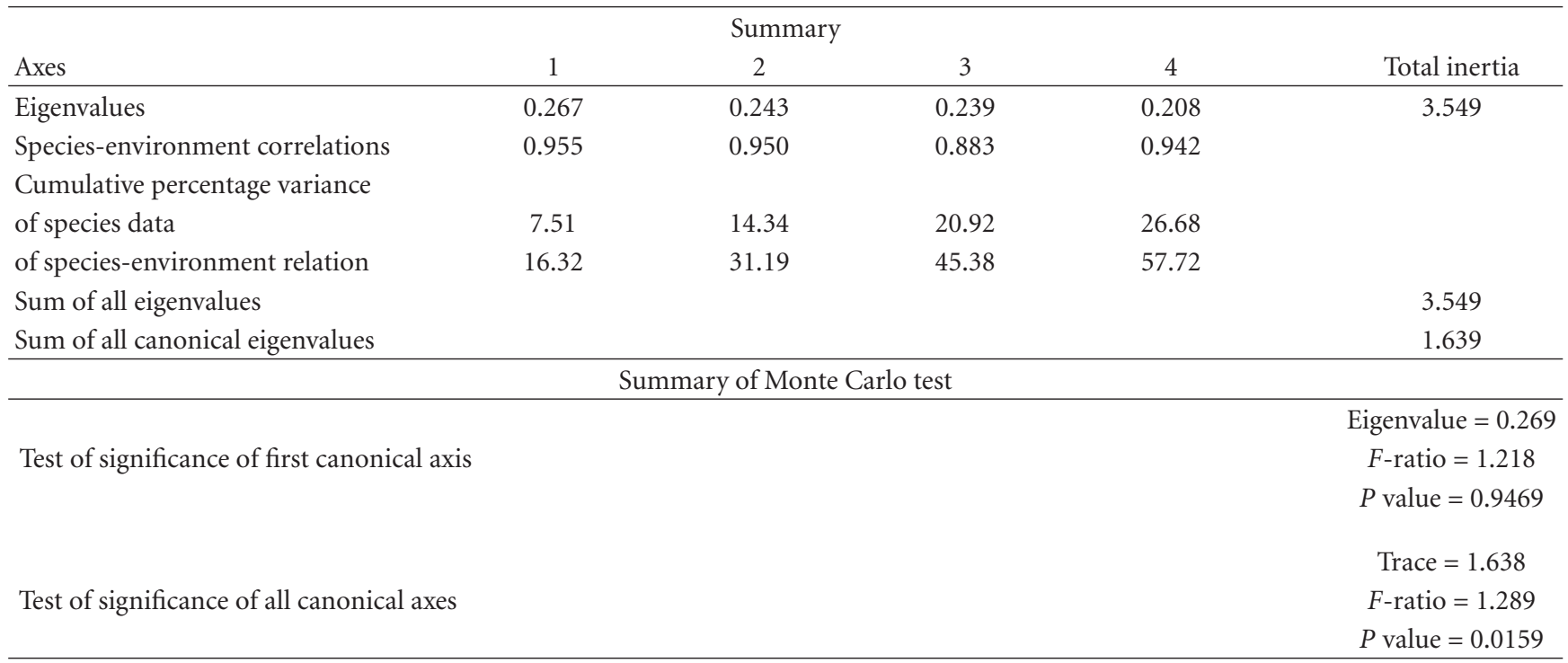

TABLE 5: Coefficient of correlations between the matrix of environmental (habitat/land-use) variables and the 4 axes of the CCA ordination scores. Different levels of significance of Spearman rank coefficients of correlation of the environmental variables with axes: ${ }^{*} P<0.05$; ${ }^{* *} P<0.01$; ${ }^{* * *} P<0.001$; ns: $P>0.05$. Correlations indicate the degree to which species occurrence and distributions correspond to changes in each variable in the environmental matrix.

\begin{tabular}{llccccc}
\hline \multicolumn{2}{l}{ Variable codes } & $\begin{array}{l}\text { Definition of environmental (habitat/land use) variables that were } \\
\text { measured concurrently with butterfly surveys }\end{array}$ & AX1 & AX2 & AX3 & AX4 \\
\hline Ag fi & Small-scale agroforestry fields with fruit tree species & $-0.025 \mathrm{~ns}$ & $-0.2264 \mathrm{~ns}$ & $0.6265^{* * *}$ & $-0.2697 \mathrm{~ns}$ \\
Pla cof & Large coffee plantations in the margins of native forest fragments & $0.158 \mathrm{~ns}$ & $0.0732 \mathrm{~ns}$ & $0.5488^{* *}$ & $0.0641 \mathrm{~ns}$ \\
Fo p & Annual/biannual crop fields in the surrounding of forest reserves & $0.1809 \mathrm{~ns}$ & $0.5947^{* *}$ & $0.4686^{*}$ & $0.2083 \mathrm{~ns}$ \\
Gr pl & Small-scale grazing fields (cows, goats, sheep) + hedgerows & $0.2799 \mathrm{~ns}$ & $0.5748^{* *}$ & $0.4771^{*}$ & $0.1021 \mathrm{~ns}$ \\
Fall & Fallows (young, old, forest and mixed fallows) & $0.521^{* *}$ & $-0.1895 \mathrm{~ns}$ & $-0.0199 \mathrm{~ns}$ & $0.4696^{*}$ \\
SS field & Small-scale monoculture/polyculture fields of annual/biannual crops & $0.3531 \mathrm{~ns}$ & $0.2615 \mathrm{~ns}$ & $0.631^{* *}$ & $-0.0049 \mathrm{~ns}$ \\
Sugar & Large sugar plantations with large water canals & $-0.1413 \mathrm{~ns}$ & $-0.0512^{*}$ & $-0.2619 \mathrm{~ns}$ & $0.4583^{*}$ \\
Sw hbt & Swampy habitats + reclaimed wetlands + stream-edges & $0.1242 \mathrm{~ns}$ & $-0.495^{*}$ & $0.5256^{* *}$ & $-0.2101 \mathrm{~ns}$ \\
Tea & Large-scale of tea plantations in vicinity of wetlands/swamps & $-0.4515^{*}$ & $-0.001 \mathrm{~ns}$ & $-0.3991^{*}$ & $0.0468 \mathrm{~ns}$ \\
Wdld & Woodlots (Eucalyptus/Pine plantations) + woodlands + grasslands & $0.0325 \mathrm{~ns}$ & $0.452^{*}$ & $0.5594^{* *}$ & $0.2514 \mathrm{~ns}$ \\
\hline
\end{tabular}

richness and density of butterflies may be attributed to monthly variations in climatic factors such as temperature and precipitation. The seasonality in butterfly species occurrence tended not to follow very closely the general patterns of rainfall in central Uganda. However, peak in butterfly species richness appeared to coincide with peaks in flowering of the most butterfly food plants.

In this study, it was observed that sites with higher cover of pasturelands, grasslands, woodlands, fallows, swamps, and stream edges had great diversity, species richness and population density compared to sites with high tree cover in their surroundings. Also, species richness was highest in study sites that were riparian forest fragments and related natural habitats (wetlands). Additionally, it was observed that study sites with higher farm management intensities were less diverse since large monoculture plantations supported less species rich butterfly communities compared to small-scale fields. The significant difference in mean species richness and diversity across sites was also accounted for by the difference in site characteristics (difference in quality of seminatural and natural habitats found within and in the vicinity of fields). The high species richness of butterflies found may be explained by the fact that mosaic of farm landscapes of central Uganda are characterized by diverse habitat types within the heterogeneously used agricultural matrix that contain a variety of host plants and breeding sites required by butterfly species belonging to different ecological groups. Additionally, strongest differences observed in species richness, abundance, and diversity were apparently related to site biophysical characteristics (see agroforests characteristics in Table 1).

The influence of site biophysical characteristics on diversity and abundance of butterflies was also supported by the ordination analysis. The presence of forest reserves, 
TABLE 6: Richness and abundance of butterflies attracted to different habitats (land uses) frequently observed in the coffee-banana farming systems of central Uganda. (Most attractive habitats were those with $>20 \%$ of weeds/crops/grass/wild plant species blooming or with buds in flowers at the time of visit.)

\begin{tabular}{|c|c|c|c|c|c|}
\hline $\begin{array}{l}\text { Foraging habitats: seminatural habitat } \\
\text { types/crop-field habitat types }\end{array}$ & Habitat Frequency & $\begin{array}{l}\text { Habitat size range } \\
\text { (ha) }\end{array}$ & $N$ & $\begin{array}{l}\text { Butterfly species }(x \\
\quad+\text { SE })\end{array}$ & $\begin{array}{l}\text { Butterfly abundance } \\
\qquad(x+\mathrm{SE})\end{array}$ \\
\hline $\begin{array}{l}\text { Field margins associated with or not termite } \\
\text { mounds and shrubs/trees/grass species }\end{array}$ & 125 & $0.05-0.5$ & 159 & $5.70 \pm 0.41 \mathrm{~d}$ & $16.91 \pm 3.52 c$ \\
\hline $\begin{array}{l}\text { Boundaries of small-scale } \\
\text { polyculture/monoculture fields }\end{array}$ & 96 & $0.01-0.05$ & 150 & $3.70 \pm 0.81 \mathrm{e}$ & $27.42 \pm 3.65 c$ \\
\hline $\begin{array}{l}\text { Forest reserves in vicinity of small-scale fields and } \\
\text { large coffee plantations }\end{array}$ & 25 & $0.5-40$ & 45 & $21.2 \pm 2.12 \mathrm{a}$ & $55.83 \pm 12.1 \mathrm{a}$ \\
\hline Woodlands, grasslands & 15 & $0.5-40$ & 30 & $16.5 \pm 1.07 b$ & $31.44 \pm 2.87 \mathrm{c}$ \\
\hline Forest plantations (Pine/Eucalyptus) & 11 & $0.5-30$ & 22 & $13.1 \pm 0.34 b$ & $24.61 \pm 0.21 c$ \\
\hline $\begin{array}{l}\text { Simple and complex traditional agroforests with } \\
\text { fruit trees }+ \text { pulse crops }+ \text { cereals + root/tuber } \\
\text { crops }\end{array}$ & 34 & $0.05-2.9$ & 119 & $14.56 \pm 1.21 b$ & $29.34 \pm 2.72 c$ \\
\hline $\begin{array}{l}\text { Agroforestry woodlots (with species like Moringa } \\
\text { sp., Sesbania sp., Leucaena sp., Calliandra sp., etc.) }\end{array}$ & 21 & $0.05-2$ & 45 & $3.12 \pm 0.55 \mathrm{e}$ & $19.55 \pm 5.61 \mathrm{c}$ \\
\hline Grazing fields, pastures, hedgerows & 67 & $0.05-10$ & 190 & $9.90 \pm 1.87 \mathrm{c}$ & $17.86 \pm 4.67 \mathrm{c}$ \\
\hline Swampy habitats, wetland-edges, streams-edges & 54 & $0.3-5$ & 89 & $17.7 \pm 2.51 b$ & $67.87 \pm 18.2 \mathrm{a}$ \\
\hline Abandoned gardens & 46 & $0.02-1$ & 258 & $10.1 \pm 1.12 \mathrm{c}$ & $41.58 \pm 7.67 b$ \\
\hline Fallows (young, old, and forest fallows) & 100 & $0.03-3$ & 356 & $16.4 \pm 1.97 b$ & $46.69 \pm 8.76 b$ \\
\hline $\begin{array}{l}\text { Herbaceous or unweeded crop fields and } \\
\text { unplowed fields }\end{array}$ & 121 & $0.02-3$ & 367 & $16.78 \pm 1.56 b$ & $36.89 \pm 1.15 c$ \\
\hline $\begin{array}{l}\text { Perennial crops (e.g., large plantations of } \\
\text { coffee/sugar cane) }\end{array}$ & 89 & $0.05-12$ & 600 & $3.92 \pm 0.23 \mathrm{e}$ & $16.32 \pm 1.11 \mathrm{c}$ \\
\hline $\begin{array}{l}\text { Perennial crops (banana) grown sole or mixed } \\
\text { with coffee and Vanilla }\end{array}$ & 65 & $0.05-15$ & 789 & $1.18 \pm 0.92 h$ & $3.268 \pm 0.25 d$ \\
\hline $\begin{array}{l}\text { Perennial tree fruit crops (avocado, mango, citrus, } \\
\text { lemon, guava, papaya, etc.) grown sole }\end{array}$ & 25 & $0.03-10$ & 98 & $3.76 \pm 0.98 \mathrm{e}$ & $16.43 \pm 1.13 c$ \\
\hline Perennial fruit crops (passion fruit) grown sole & 12 & $0.01-3$ & 22 & $3.12 \pm 1.13 \mathrm{e}$ & $15.65 \pm 1.59 c$ \\
\hline $\begin{array}{l}\text { Homegardens of annual vegetables (pumpkin, } \\
\text { watermelon, cleome, etc.) }\end{array}$ & 48 & $0.01-0.5$ & 79 & $4.62 \pm 0.47 d$ & $25.78 \pm 1.43 c$ \\
\hline $\begin{array}{l}\text { Annual commercial/cash vegetable crops (tomato, } \\
\text { egg plant, pepper) grown sole or mixed }\end{array}$ & 38 & $0.02-6$ & 121 & $2.11 \pm 0.95 f$ & $10.21 \pm 2.35 \mathrm{~d}$ \\
\hline $\begin{array}{l}\text { Annual commercial/cash crops (sim-sim, sun } \\
\text { flower) grown sole or mixed with legumes }\end{array}$ & 15 & $0.02-10$ & 48 & $2.12 \pm 0.88 \mathrm{f}$ & $13.59 \pm 2.45 \mathrm{~d}$ \\
\hline $\begin{array}{l}\text { Annual cereals mixed with pulse crops } \\
\text { (beans,cowpea, greengram, soybean): Maize }\end{array}$ & 61 & $0.04-7$ & 421 & $2.13 \pm 0.81 f$ & $5.12 \pm 0.59 \mathrm{e}$ \\
\hline $\begin{array}{l}\text { Annual cereals mixed with pulse crops: } \\
\text { sorghum/millet }\end{array}$ & 19 & $0.03-6$ & 259 & $3.17 \pm 0.97 \mathrm{e}$ & $11.67 \pm 2.11 \mathrm{~d}$ \\
\hline $\begin{array}{l}\text { Annual cereals (maize, sorghum, millet) mixed } \\
\text { with pulse crops(beans, groundnut): Beans }\end{array}$ & 41 & $0.02-5$ & 342 & $2.43 \pm 0.31 f$ & $9.625 \pm 2.61 d$ \\
\hline $\begin{array}{l}\text { Annual cereals mixed with pulse crops: } \\
\text { Groundnut, cowpeas }\end{array}$ & 26 & $0.02-6$ & 403 & $1.23 \pm 0.34 \mathrm{~h}$ & $4.624 \pm 0.54 \mathrm{e}$ \\
\hline Bi-annual crops (cassava) & 26 & $0.02-8$ & 198 & $1.12 \pm 0.16 h$ & $4.232 \pm 0.98 \mathrm{e}$ \\
\hline Annual crops (sweet potato) & 51 & $0.02-3$ & 400 & $4.32 \pm 0.67 d$ & $8.963 \pm 2.42 \mathrm{~d}$ \\
\hline Annual cereals (rice) & 16 & $0.05-15$ & 55 & $1.13 \pm 0.13 \mathrm{~h}$ & $4.651 \pm 0.87 \mathrm{e}$ \\
\hline Annual crops (Irish potato) & 28 & $0.05-12$ & 43 & $2.45 \pm 0.41 \mathrm{~h}$ & $12.34 \pm 2.76 \mathrm{~d}$ \\
\hline
\end{tabular}

Habitat frequency: number of observation cases or number of times the habitat type was encountered across all 26 study sites and all sampling rounds. Habitat size range (ha): the data show the minimum and the maximum size of the type of habitat encountered during butterfly faunistic surveys.

$N$ : number of samples (butterfly species and individuals) recorded in five sampling rounds across the 26 study sites in 2006.

Within columns, different letters show significant differences of the means at $P=0.05$ according to LSD test performed after Kruskall Wallis-ANOVA test indicated that the habitat type was significant $(P<0.001)$ for the number of species and individuals attracted. 
TABLE 7: Effect of the farm intensity management system on the richness, abundance, and diversity of butterfly found in the coffee-banana farming system in central Uganda.

\begin{tabular}{lccc}
\hline Farm management systems & $\begin{array}{c}\text { Species richness } \\
\text { (number species/site) }\end{array}$ & $\begin{array}{c}\text { Abundance } \\
\text { (individuals/site) }\end{array}$ & $\begin{array}{c}\text { Diversity } \\
\text { (Shannon-diversity index: } H^{\prime} \text { ) }\end{array}$ \\
\hline Modernized (large-scale fields) & $14.21 \pm 2.73 \mathrm{~b}$ & $330.2 \pm 79.55 \mathrm{~b}$ & $2.28 \pm 0.81 \mathrm{~b}$ \\
Traditional (small-scale fields) & $19.95 \pm 4.41 \mathrm{a}$ & $685.7 \pm 231.47 \mathrm{a}$ & $2.89 \pm 1.59 \mathrm{a}$ \\
\hline
\end{tabular}

Within each column, means followed by the same letters are not significantly different at 5\%, LSD test.

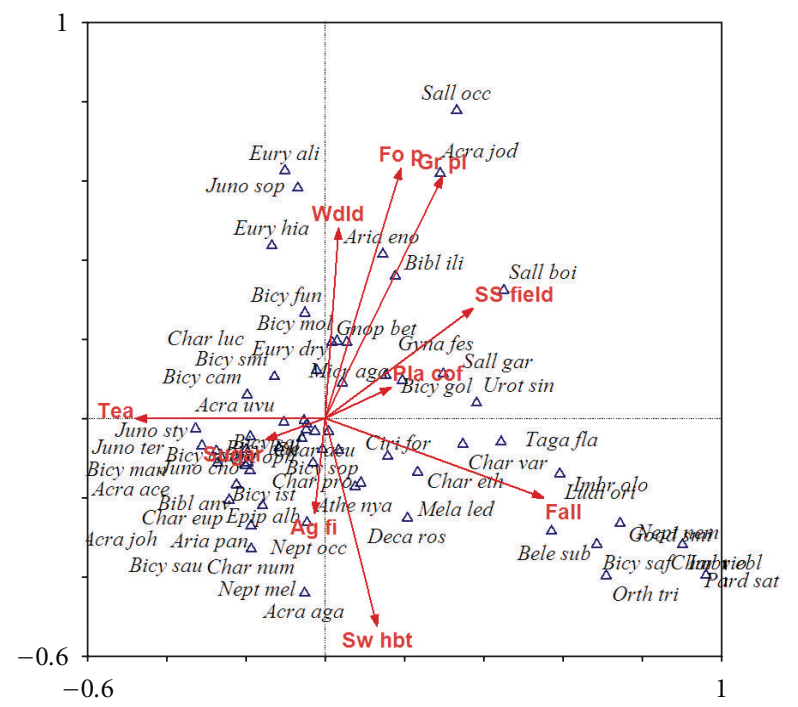

FIgURE 4: Canonical correspondence analysis (CCA) ordination diagram biplot showing correlation between butterfly specie (Acra joh, Mela led, and Bicy sau are short names of specie) and the environmental variables (codes: solid line vector "Tea," "Sw hbt," "Fall," etc.).

woodlands, fallows, hedgerows, woodlots (pine/eucalyptus forest plantations) in the vicinity of fields were environmental factors that appeared to be more important and primary determining the occurrence of foraging butterfly species composition. The ordination analysis demonstrated that the occurrence of different butterfly species in the farmland was strongly positively association with availability of natural and seminatural habitats found in the surrounding of fields. Thus, seminatural and natural habitats are among environmental factors playing key roles in shaping butterfly communities potentially delivering pollination services to wild and cultivated plants in the coffee-banana agroforestry systems of central Uganda.

4.2. Butterfly Assemblages in Tropical Agroforestry Systems. In this study, farmlands were hypothesized not to be suitable habitats for diverse butterfly communities in central Uganda. In contrast to expectations, the results indicated that farmlands of central Uganda supported a rich butterfly fauna and that there were significant $(P<0.05)$ variation in species richness, abundance and diversity among the 26 sites studied. Small-scale polyculture fields and other traditional complex agroforestry systems that were established in the vicinity of forests fragments or traditional simple agroforestry systems with a high cover of seminatural habitats (fallows, swamps, hedgerows) supported more species and individuals of butterflies than other typical cropping systems. These important agroforestry systems supported also a high number of species of butterfly food plants. Several butterfly food plants species were recorded in a previous study [7].

In this study it was observed that farmlands of central Uganda supported species rich-butterfly faunas that are highly seasonal. The high number of butterfly species and individuals recorded in small scale polycultures indicated that a certain number of wild and cultivated plant species were receiving Lepidoptera pollination services of high quality. In other words, several butterfly species occurring in the farmland of central Uganda may be efficient pollinator species on many cultivated and wild plant species (subspecies, land races, genotypes, varieties). In fact, some butterfly species in the Lycaenidae, Nymphalidae family were frequently recorded collecting floral resources on several weeds/crops in bloom that were found in different small scale field where cereals and legumes were mixed. In farmlands of central Uganda, butterflies have been observed being engaged in different activities (perching, flying, mating, foraging, and oviposition). With field experience, some butterfly species were observed making long visits on crop flowers such as cowpeas, beans, groundnut, and egg plants. They made their visits to crop flowers mainly between 9 h00 and 11 h00 and between 14 h00 and 16 h00 (Figure 5).

In central Uganda, Catopsilia florella, Bicyclus safitza, Junonia Sophia, and Acraea acerata were especially abundant while a much larger number of species were relatively rare ( $>60 \%$ of species recorded), reflecting patterns expected from tropical environments. These species appeared to be well adapted for survival in farmlands of central Uganda. They are well known to be abundant in grassland habitats in Uganda [7]. They are probably typical farmland species as no reports have ever mentioned them among the dominant species of forest ecosystems in Uganda. In addition, Catopsilia florella and Acraea acerata have been reported by local farmers to be pests, although scientific evidence indicates that only A. acerata is a pest of crop species such as sweetpotato [30]. Similar observations were reported by Fernández-Hernández [31] in Cuba.

Farmland butterflies are believed to pollinate many wild and cultivated plant species. Pollination activities of some species are well known. Continuous observations are needed in the future in order to determine the pollination efficiency 


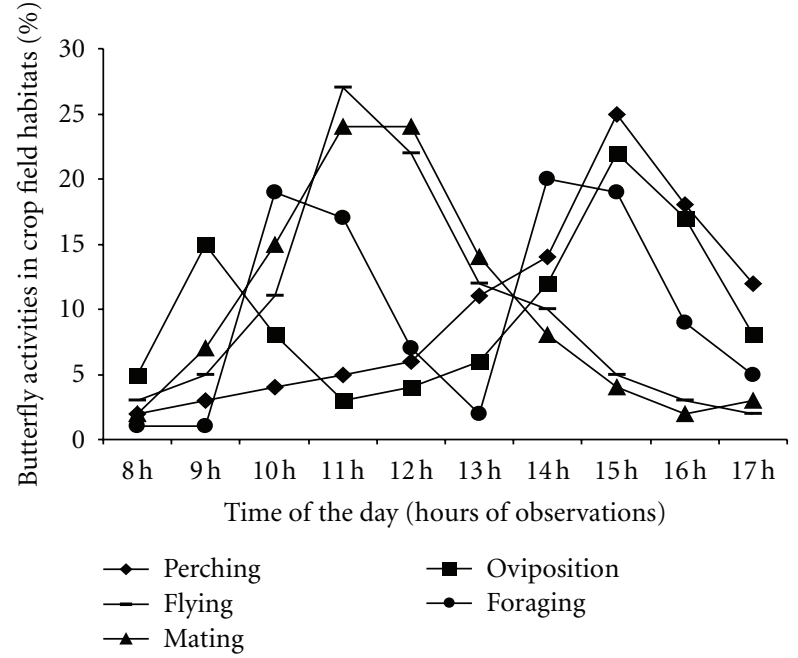

FIGURE 5: Distribution of the daily activities of few butterfly species that were monitored when visiting crop field habitasts in centrtal Uganda in 2006-preliminary results. With author experience, observations were recorded $(N=679$ observations conducted on 5 butterfly species).

of all butterfly species on wild and cultivated plant species. This may help in development of strategies for their conservation and management within farmland habitats. The functional status (as pest or as pollinator) of Catopsilia florella remains largely unknown. This species disperses locally and in this study, the species was observed foraging frequently on both flowering herbs/weeds and on some crop species.

In central Uganda, farmlands were found to be visited by a high number of species of "forest dependent species" (mainly Nymphalidae). This finding contrasts with observations of Fermon et al. [32] who found that the majority of Nymphalidae species were avoiding farmlands located in the vicinity of forest habitats. Fermon et al. [32] stressed that only a few specialist nymphalid species could visit pine/eucalyptus plantations adjacent to the natural forests in Bossematie zone (Cote D'ivoire). The main reason for central Uganda to support high butterfly diversity dominated by Nymphalidae is the presence of forest patches within agricultural matrices, making central Uganda to appear more like "small degraded forests than just typical agricultural mosaic landscape" [7].

4.3. Patterns of Butterfly Diversity and Abundance in Farmland Habitats. In this study, a great variation in species richness and density among the 26 study sites was observed. The most species-rich site contained more than four times the number of species as the poorest site overall. The highest species richness, population density, and diversity of butterflies were found to be associated with study sites that are riparian forest reserves (see Table 1).

Much as factors governing butterfly community structure and composition in farmlands are not fully known, this result indicates that forest vegetation play a significant role in the distribution, diversity, and density of butterfly species in farmlands of central Uganda. A possible explanation is that butterflies visit farmlands for supplemental nectar resources not found in the adjacent forest ecosystems.

In East Africa, farmlands are expected to be populated by a small number of butterfly species, generally 5 to 50 represented by a relatively high number of individuals. While in this study it was observed that a high number of forestdependent species ( $40 \%$ of 331 species recorded), including fruit-feeding species, that visited crop fields located in the adjacent; a recent study conducted in Ghana found that very few ( $<10 \%$ of 90 species recorded) fruit-feeding species occurred in the farm bush savannah [33].

Several studies indicate that farmland habitats support poor communities of butterflies [14, 34-36]. However, few studies have highlighted similar findings to those obtained in central Uganda. For example, in Vietnam, Lien and Yuan [37] recorded a higher diversity of butterflies in agricultural habitats $[28,37]$. Similar observations were recorded by Lien and Yuan [37] in Costa Rica where high species richness of butterfly was recorded in coffee plantations as compared to adjacent forest remnant fields. In addition, Kitahara [21] recorded higher species richness and diversity in agricultural landscapes in Japan compared to forest fragments. These previous studies show that elsewhere (out of sub-Sahara Africa) farmland habitats can be important for butterflies comparable to forest ecosystems as it was observed in central found in Uganda.

In conclusion, the overall objective of this study was to provide information on butterfly communities to conservation planners and policy makers in central Uganda. The results show that the species diversity of butterfly in the coffee-banana agroforestry systems of central Uganda is much higher than expected. Butterflies visited a variety of habitats and land uses, some of them were better than others to harbor butterflies. Important habitats for farmland butterflies included woodlands, fallows, hedgerows, swampy habitats, abandoned gardens, and homegardens.

Findings from this study indicated that agricultural field sites that were riparian forest fragments/wetlands had the highest species richness and population density of butterflies compared to field sites established far from natural habitats (forests, wetlands). They might support the concept of corridors across large agricultural areas.

Higher forest cover within landscapes had a major positive influence on butterfly biodiversity in agricultural landscapes and related human-modified landscapes in central Uganda.

Although data was collected during five rounds (knowing that five samples may not be enough for robust statistical analysis) conducted in only one year (2006), this study supplied valuable information about the diversity of butterflies in coffee-banana agroforestry areas in central Uganda. The research findings indicated that local coffee-banana agroforestry systems are valuable for butterfly conservation.

A high number of species and individuals were recorded in small scale polycultures. This indicated that some wild and cultivated plant species were potentially receiving pollination 
services of high quality from different butterfly species. There is a need to develop appropriate strategies to conserve coffee-banana agroforests to maintain butterfly communities delivering pollination services to crops and wild plants within and nearby crops habitats. The conservation of remnant forest patches through restoration of different habitats, protection from degradation and improvement of the connectivity to larger forest patches and related manmade forest plantations (woodlots, forest plantations) needs to be prioritized by policy makers and land-use planners. Their protection from degradation should be a priority for decision makers since these forest support a rich community of butterflies potentially delivering pollination services to cultivated plants on which human beings depend on for their livelihoods.

Farmers are advised to adopt landscape management and farming practices that are friendly to butterfly species pollinating some of their crops such as practices that can increase high on-farm cover (\%) of different types of land uses (polycultures of cereals mixed to legume crops, traditional simple and complex agroforestry systems), of seminatural features (hedgerows, fallows, grasslands, pasturelands, swampy habitats, stream-edges) and related habitats (forest patches, forest fallows, woodlots of eucalyptus, pines) or farming practices that protect and increase the area covered by noncrop habitats within farmed landscape to serve as "butterfly reservoirs." Such uncultivated areas can also support larval host plants and various nectarine plant species that may attract different adult butterfly species in the farmlands.

Farmers are recommended to invest in the protection of habitats to offer habitat and nectar sources to butterflies in the farmland. The protection of natural and seminatural habitats in the coffee-banana farming system may be of different uses: serving as source of butterflies for a butterfly farm. The coffee-banana farming system may also be used as tourist place to see common farmland butterflies species. More practically, they need protection since preliminary observations indicated that some cultivated crops were receiving pollination services [7] from some butterfly species.

Farm management regime had an impact of butterfly diversity such as small scale farms supported highly diverse butterfly communities than did modernize large plantations. Therefore, farmers are encouraged to adopt polyculture cropping systems integrated to good management of floral resources in the margins of fields.

To provide contributions to the conservation of butterfly fauna in agricultural landscapes in Uganda, it is important to determine the pollination efficiency of different butterfly species. The recommended correct sampling method to bring appropriate result is transect count. This method may be a suitable method to assess the diversity of butterfly communities delivering pollination services to wild and cultivated crops in different farmland habitats of central Uganda. The method is recommended to be used for monitoring butterflies, as well as assessing impact of habitat disturbance on community diversity of butterflies. Banana-bait trap method may be recommended for assessing/monitoring fruit feeding community in the area while hand net may be used when aiming at detecting rapidly a high number of species in the habitat.

\section{Acknowledgments}

The author is very grateful to Darwin Initiative for funding this study. Also, he is very grateful to farmers for offering their farms for conducting butterfly sampling. The valuable inputs in this work from Professor Philip Nyeko (Makerere University Uganda) and Professor Simon Potts (University of Reading UK) (scientific supervisors) are acknowledged. The author owes his deepest gratitude to Dr. Phil Atkinson (British Trust Ornithology, UK), Dr. Juliet Vickery (RSPBCambridge-UG), and Professor Derek Pomeroy (project leaders and scientific advisors) for their financial support. Mr. Maurice Mutabazi is thanked for his assistance in the field. The author acknowledges the warm and kind collaboration of Professor Frank Kansiime (Deputy-Principal of Makerere University College of Agricultural and Environmental sciences) who facilitated administration issues related to field sampling.

\section{References}

[1] S. Kumar, S. E. Simonson, and T. J. Stohlgren, "Effects of spatial heterogeneity on butterfly species richness in Rocky Mountain National Park, CO, USA," Biodiversity and Conservation, vol. 18, no. 3, pp. 739-763, 2009.

[2] C. Kremen, "Biological inventory using target taxa: a case study of the butterflies of Madagascar," Ecological Applications, vol. 4, no. 3, pp. 407-422, 1994.

[3] C. Kremen, "Assessing the indicator properties of species assemblages for natural areas monitoring," Ecological Applications, vol. 2, no. 2, pp. 203-217, 1992.

[4] B. C. Schmidt and J. Roland, "Moth diversity in a fragmented habitat: importance of functional groups and landscape scale in the boreal forest," Annals of the Entomological Society of America, vol. 99, no. 6, pp. 1110-1120, 2006.

[5] M. O. Marchiori and H. P. Romanowski, "Species composition and diel variation of a butterfly taxocene (Lepidoptera, Papilionoidea and Hersperioidea) in a restinga forest at Itapuã State Park, Rio Grande do Sul, Brazil," Revista Brasileira de Zoologia, vol. 23, no. 2, pp. 443-454, 2006.

[6] K. S. Summerville, E. H. Metzler, and T. O. Crist, "Diversity of Lepidoptera in Ohio forests at local and regional scales: how heterogeneous is the fauna?" Annals of the Entomological Society of America, vol. 94, no. 4, pp. 583-591, 2001.

[7] T. M. B. Munyuli, Pollinator biodiversity and economic value of pollination services in Uganda [Ph.D. Dissertation], Makerere University, Kampala, Uganda, 2010.

[8] L. Boriani, G. Burgio, M. Marini, and M. Genghini, "Faunistic study on butterflies collected in Northern Italy rural landscape," Bulletin of Insectology, vol. 58, no. 1, pp. 49-56, 2005.

[9] G. Tumuhimbise, M. J. N. Okwakol, and T. N. Kangwagye, "Species diversity of swallowtail butterflies (Papilionidae: Lepidoptera) in North Maramagambo Forest," African Journal of Ecology, vol. 39, no. 1, pp. 113-115, 2001.

[10] P. C. Howard, T. R. B. Davenport, F. W. Kigenyi et al., "Protected area planning in the tropics: Uganda's national system of forest nature reserves," Conservation Biology, vol. 14, no. 3, pp. 858-875, 2000. 
[11] H. Tushabe, J. Kalema, A. Byaruhanga et al., "A nationwide assessment of the biodiversity value of Uganda's important bird areas network," Conservation Biology, vol. 20, no. 1, pp. 85-99, 2006.

[12] F. Molleman, A. Kop, P. M. Brakefield, P. J. De Vries, and B. J. Zwaan, "Vertical and temporal patterns of biodiversity of fruit-feeding butterflies in a tropical forest in Uganda," Biodiversity and Conservation, vol. 15, no. 1, pp. 107-121, 2006.

[13] J. D. Davis, S. D. Hendrix, D. M. Debinski, and C. J. Hemsley, "Butterfly, bee and forb community composition and crosstaxon incongruence in tallgrass prairie fragments," Journal of Insect Conservation, vol. 12, no. 1, pp. 69-79, 2008.

[14] E. Fitzherbert, T. Gardner, T. R. B. Davenport, and T. Caro, "Butterfly species richness and abundance in the Katavi ecosystem of western Tanzania," African Journal of Ecology, vol. 44, no. 3, pp. 353-362, 2006.

[15] D. Kuefler, N. M. Haddad, S. Hall, B. Hudgens, B. Bartel, and E. Hoffman, "Distribution, population structure and habitat use of the endangered Saint Francis Satyr butterfly, Neonympha mitchellii francisci," American Midland Naturalist, vol. 159, no. 2, pp. 298-320, 2008.

[16] E. Pollard and T.G. Yates, Monitoring Butterflies for Ecology and Conservation, vol. 1, Chapman and Hall, London, UK, 1993.

[17] M. J. Coe, N.C. McWilliams, G.N. Stone, M.J. Packer, and "Mkomazi: The Ecology, Biodiversity and Conservation of a Tanzanian Savannah, Royal geographical Society (with the Institute of British Geographers), London, UK, 1999.

[18] M. Kitahara and K. Sei, "A comparison of the diversity and structure of butterfly communities in semi-natural and human-modified grassland habitats at the foot of Mt. Fuji, central Japan," Biodiversity and Conservation, vol. 10, no. 3, pp. 331-351, 2001.

[19] P. Akite, "Effects of anthropogenic disturbances on the diversity and composition of the butterfly fauna of sites in the Sango Bay and Iriiri areas, Uganda: implications for conservation," African Journal of Ecology, vol. 46, no. 1, pp. 3-13, 2008.

[20] D. F. Owen, Tropical Butterflies. The Ecology and Behavior of Butterflies in the Tropics with Special Reference to African Species, Oxford Calendenon Press, Oxford, UK, 1971.

[21] M. Kitahara, M. Yumoto, and T. Kobayashi, "Relationship of butterfly diversity with nectar plant species richness in and around the Aokigahara primary woodland of Mount Fuji, central Japan," Biodiversity and Conservation, vol. 17, no. 11, pp. 2713-2734, 2008.

[22] T. B. Larsen, The Butterflies of Kenya and Their Natural History, Oxford University Press, Oxford, UK, 1996.

[23] T. B. Larsen, "Forest butterflies in West Africa have resisted extinction... so far (Lepidoptera: Papilionoidea and Hesperioidea)," Biodiversity and Conservation, vol. 17, no. 12, pp. 2833-2847, 2008.

[24] N. Carder and L. Tindimubona, Butterflies of Uganda. A Field Guide to Butterflies and Silk Moths. A Collection of the Uganda Society, The Uganda society, Kampala, Uganda, 2002.

[25] T. R. B. Davenport, The Butterflies of Uganda-An Annotated Checklist, The Forest Department, Kampala, Uganda, 1996.

[26] M. L. T. Buschini and T. D. Woiski, "Alpha-beta diversity in trap-nesting wasps (Hymenoptera: Aculeata) in Southern Brazil," Acta Zoologica, vol. 89, no. 4, pp. 351-358, 2008.

[27] A. E. Magurran, Measuring Biology Diversity, Blackwell , Oxford, UK, 2004.

[28] N. J. Gotelli and R. K. Colwell, "Quantifying biodiversity: procedures and pitfalls in the measurement and comparison of species richness," Ecology Letters, vol. 4, no. 4, pp. 379-391, 2001.
[29] C. J. F. Ter Braak and P. Šmilauer, Canoco for Windows Version 4.5, Centre for Biometry Wageningen, Wageningen, The Netherlands, 2002.

[30] T. M. B. Munyuli, "Yield loss evaluation of sweet potato due to leaf caterpillar, Acraea acerata, (Lepidoptera: Nymphalidae)," African Potato Association Conference Proceedings, vol. 5, pp. 233-236, 2000.

[31] D. M. Fernández-Hernández, "Butterflies of the agricultural experiment station of tropical roots and tubers, and santa ana, camagüey, Cuba: an annotated list," Acta Zoológica Mexicana, vol. 23, no. 2, pp. 43-75, 2007.

[32] H. Fermon, M. Waltert, T. B. Larsen, U. Dall'Asta, and M. Mühlenberg, "Effects of forest management on diversity and abundance of fruit-feeding nymphalid butterflies in southeastern Cote d'Ivoire," Journal of Insect Conservation, vol. 4, no. 3, pp. 173-189, 2000.

[33] J. P. Elbers and J. L. Bossart, "Occurrences of forest butterflies in the farm bush savannah outside a forest reserve in Ghana, West Africa," International Journal of Tropical Insect Science, vol. 29, no. 3, pp. 141-150, 2009.

[34] C. A. M. Van Swaay, P. Nowicki, J. Settele, and A. J. Van Strien, "Butterfly monitoring in Europe: methods, applications and perspectives," Biodiversity and Conservation, vol. 17, no. 14, pp. 3455-3469, 2008.

[35] L. V. Vu, "Diversity and similarity of butterfly communities in five different habitat types at Tam Dao National Park, Vietnam," Journal of Zoology, vol. 277, no. 1, pp. 15-22, 2009.

[36] J. Barlow, W. L. Overal, I. S. Araujo, T. A. Gardner, and C. A. Peres, "The value of primary, secondary and plantation forests for fruit-feeding butterflies in the Brazilian Amazon," Journal of Applied Ecology, vol. 44, no. 5, pp. 1001-1012, 2007.

[37] V. Van Lien and D. Yuan, "The differences of butterfly (Lepidoptera, Papilionoidea) communities in habitats with various degrees of disturbance and altitudes in tropical forests of Vietnam," Biodiversity and Conservation, vol. 12, no. 6, pp. 1099-1111, 2003. 

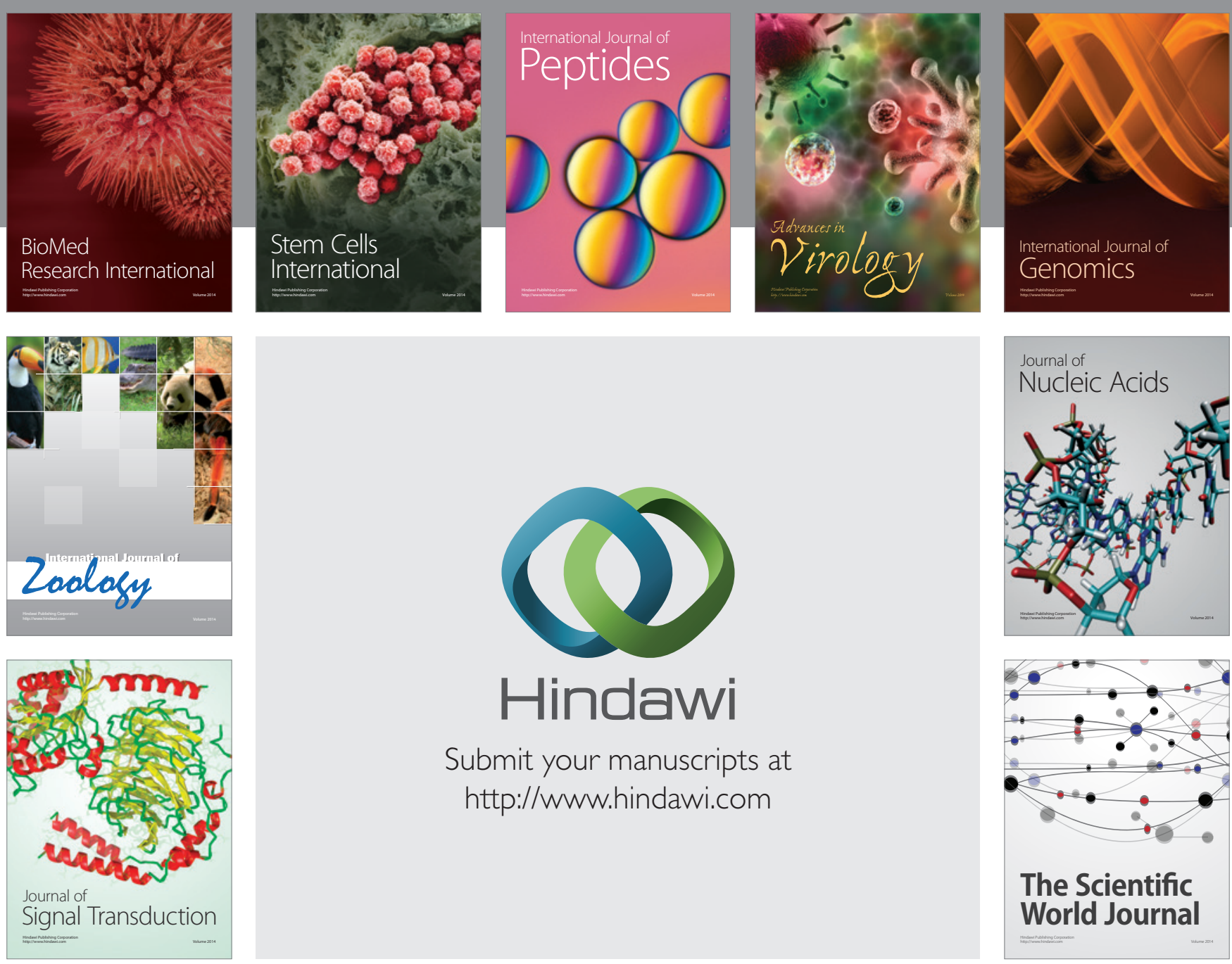

Submit your manuscripts at

http://www.hindawi.com
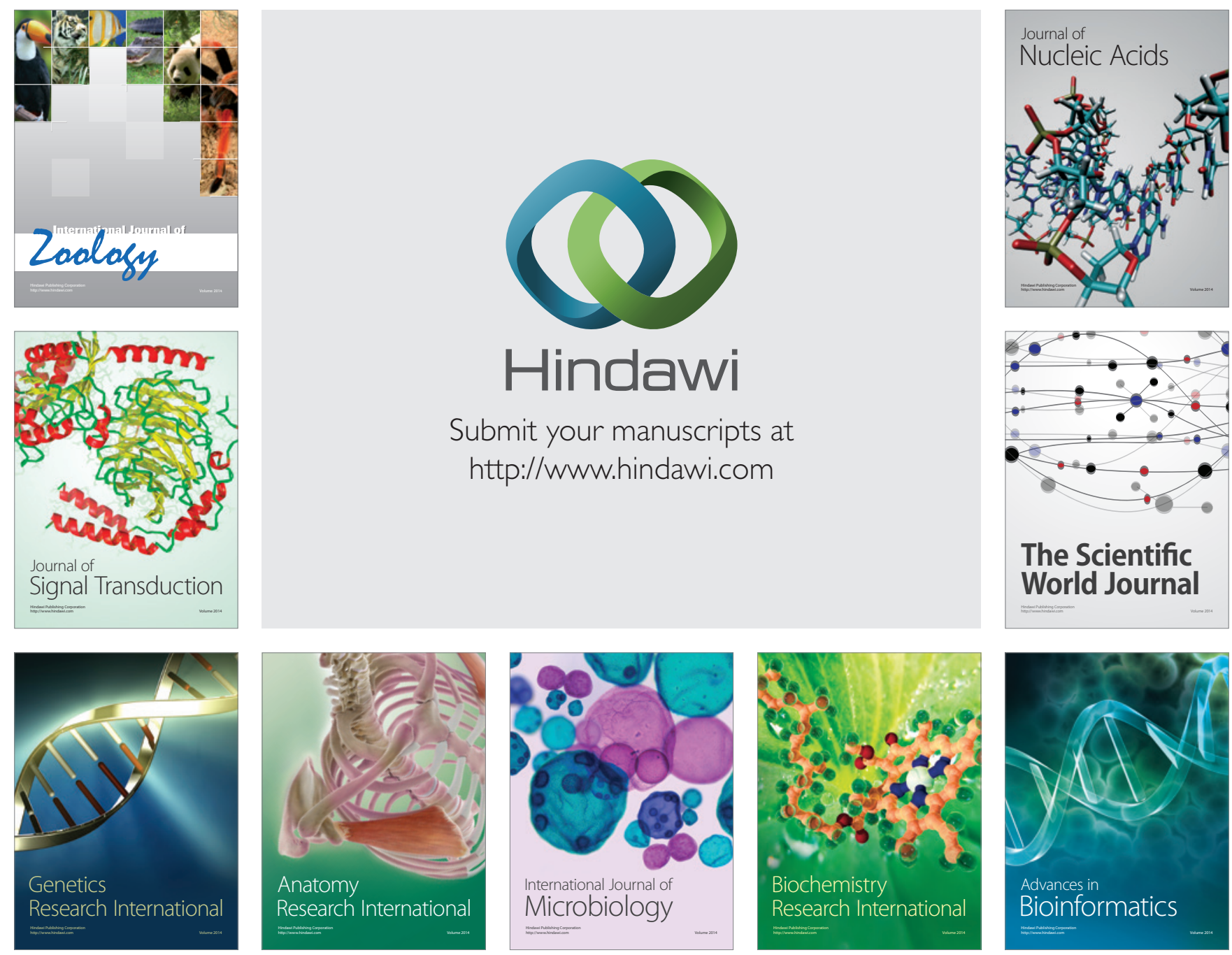

The Scientific World Journal
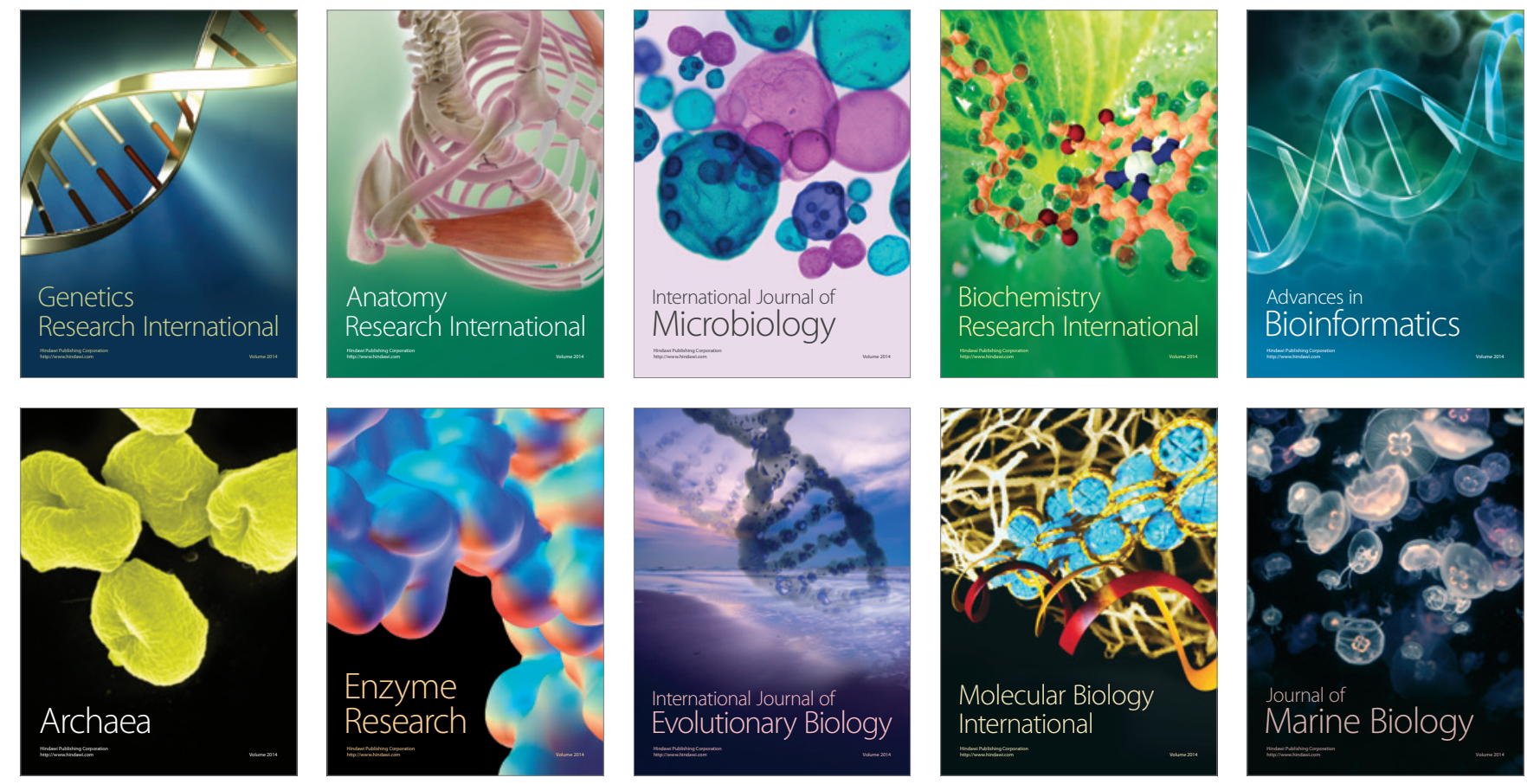